\title{
HILBERT SCHEME OF RATIONAL CUBIC CURVES VIA STABLE MAPS
}

\author{
KIRYONG CHUNG AND YOUNG-HOON KIEM
}

\begin{abstract}
The space of smooth rational cubic curves in projective space $\mathbb{P}^{r}$ $(r \geq 3)$ is a smooth quasi-projective variety, which gives us an open subset of the corresponding Hilbert scheme, the moduli space of stable maps, or the moduli space of stable sheaves. By taking its closure, we obtain three compactifications $\mathbf{H}, \mathbf{M}$, and $\mathbf{S}$ respectively. In this paper, we compare these compactifications. First, we prove that $\mathbf{H}$ is the blow-up of $\mathbf{S}$ along a smooth subvariety which is the locus of stable sheaves which are planar (i.e. support is contained in a plane). Next we prove that $\mathbf{S}$ is obtained from $\mathbf{M}$ by three blow-ups followed by three blow-downs and the centers are described explicitly. Using this, we calculate the cohomology of $\mathbf{S}$.
\end{abstract}

\section{INTRODUCTION}

Let $\mathbf{X}_{0}$ be the space of smooth rational curves of degree $d$ in $\mathbb{P}^{r}$. It is easy to see that $\mathbf{X}_{0}$ is a smooth quasi-projective variety of dimension $(r+1)(d+1)-4$. From moduli theoretic point of view, the following questions are quite natural.

(1) Does $\mathbf{X}_{0}$ admit a moduli theoretic compactification?

(2) If there are more than one such compactifications, what are the relationships among them?

(3) Can we calculate the differences of intersection numbers of cycles coming from geometric conditions?

For the first question, there are several well-known compactifications as we will review below. The purpose of this paper is to provide answers to the second question for $d=3$. Note that the second question is trivial when $d=1$ because $X_{0}$ is compact. The case where $d=2$ has been worked out in [10]: The moduli space of stable maps $\mathcal{M}_{0}\left(\mathbb{P}^{r}, 2\right)$ is Kirwan's partial desingularization of the quasi-map space $\mathbb{P}\left(\operatorname{Sym}^{2}\left(\mathbb{C}^{2}\right) \otimes \mathbb{C}^{r+1}\right) / / \operatorname{SL}(2)$ and the Hilbert scheme $\mathcal{H} i b^{2 m+1}\left(\mathbb{P}^{r}\right)$ is obtained from $\mathcal{M}_{0}\left(\mathbb{P}^{r}, 2\right)$ by a blow-up followed by a blow-down. The third question is related to the problem of comparing various curve counting invariants, such as the Gromov-Witten invariant, Donaldson-Thomas invariant and PandharipandeThomas invariant. A successful comparison of these curve counting invariants may be achieved if the second question is answered in a satisfactory fashion. For instance, if we can describe the birational maps between two different compactifications of $\mathbf{X}_{0}$ in terms of blow-ups and -downs whose centers are themselves moduli spaces for lower degree curves, then it is quite plausible that the differences of the curve counting invariants may be expressed as inductive formulae. In this paper we first review several natural moduli theoretic compactifications and then compare these compactifications in terms of explicit blow-ups and -downs.

Partially supported by KOSEF grant R01-2007-000-20064-0. 
In $\S 2$, we review several natural moduli theoretic compacticiations of $\mathbf{X}_{0}$. The first compactification comes from geometric invariant theory (GIT). A smooth rational cubic curve is given by a map $f: \mathbb{P}^{1} \rightarrow \mathbb{P}^{r}$. If we choose homogeneous coordinates of $\mathbb{P}^{1}$ and $\mathbb{P}^{r}, f$ is given by an $(r+1)$-tuple of homogeneous polynomials of degree $d$ in two variables $z_{0}, z_{1}$. To remove the dependency on the choice of homogeneous coordinates, we have to take the quotient by the action of $\operatorname{Aut}\left(\mathbb{P}^{1}\right)=\operatorname{PGL}(2)$. Hence, we obtain a compactification by GIT quotient, often called the quasi-map space

$$
\mathbf{X}=\mathbb{P}\left(\operatorname{Sym}^{3}\left(\mathbb{C}^{2}\right) \otimes \mathbb{C}^{\mathrm{r}+1}\right) / / \operatorname{SL}(2) .
$$

The strength of this compactification $\mathbf{X}$ is that it is easy to calculate the cohomology ring or Chow ring or K-groups by using the equivariant Morse theory, or the AtiyahBott-Kirwan theory [12]. The weakness of $\mathbf{X}$ is that the boundary points do not have natural geometric meaning.

The second compactification is obtained from the Hilbert scheme. We have the obvious embedding $\mathbf{X}_{0} \hookrightarrow \mathcal{H}$ ilb ${ }^{3 m+1}\left(\mathbb{P}^{r}\right)$ of $\mathbf{X}_{0}$ into the Hilbert scheme of closed subschemes with Hilbert polynomial $3 m+1$. This turns out to be an open immersion and by taking its closure we obtain a compactification $\mathbf{H}$ of $\mathbf{X}_{0}$, which we call the Hilbert compactificaiton.

The third compactification comes from Kontsevich's moduli space of stable maps. A stable map is a morphism of a connected nodal curve $f: C \rightarrow \mathbb{P}^{r}$ with finite automorphism group. Let $\mathcal{M}_{0}\left(\mathbb{P}^{r}, d\right)$ denote the moduli space of stable maps of arithmetic genus 0 and degree $\mathrm{d}$. It is well-known that this is an irreducible normal projective scheme. The obvious inclusion $\mathbf{X}_{0} \hookrightarrow \mathcal{M}_{0}\left(\mathbb{P}^{r}, 3\right)$ is an open immersion and $\mathbf{M}=\mathcal{M}_{0}\left(\mathbb{P}^{r}, 3\right)$ is a compactification of $\mathbf{X}_{0}$, which we call the Kontsevich compactification. In [11], we proved that $\mathbf{M}$ is obtained from $\mathbf{X}$ by three blowups and two blow-downs and the blow-up/-down centers are explicitly described in terms of moduli spaces of stable maps of degrees 1 and 2.

Theorem 1.1. [11] The birational map $\mathbf{X} \rightarrow \mathbf{M}$ is the composition of three blow-ups followed by two blow-downs. The blow-up centers are respectively, $\mathbb{P}^{r}$, $\mathcal{M}_{0,2}\left(\mathbb{P}^{r}, 1\right) / S_{2}$ (where $S_{2}$ interchanges the two marked points) and the blow-up of $\mathcal{M}_{0,1}\left(\mathbb{P}^{r}, 2\right)$ along the locus of three irreducible components. The centers of the blow-downs are respectively the $\mathrm{S}_{2}$-quotient of a $\left(\mathbb{P}^{r}\right)^{2}$-bundle on $\mathcal{M}_{0,2}\left(\mathbb{P}^{r}, 1\right)$ and a $\left(\mathbb{P}^{r-1}\right)^{3} / \mathrm{S}_{3}$-bundle on $\mathbb{P}^{r}$. Here $\mathcal{M}_{0, k}\left(\mathbb{P}^{r}, \mathrm{~d}\right)$ denotes the moduli space of stable maps of genus 0 and degree $\mathrm{d}$ with $\mathrm{k}$ marked points.

The following diagram explains how we get $\mathbf{M}$ from $\mathbf{X}$ by explicit blow-ups and -downs.

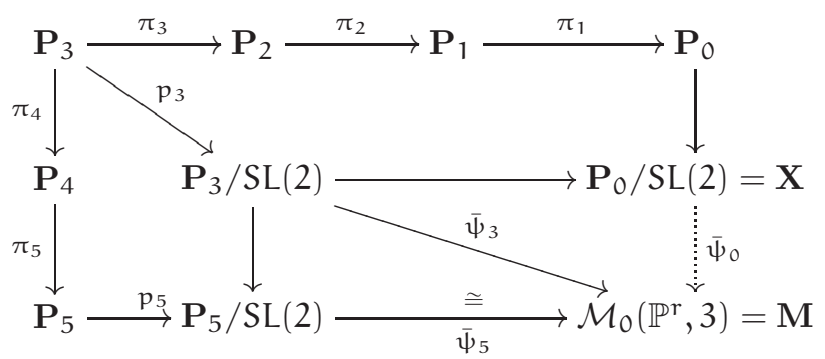


Here $\mathbf{P}_{0}$ denotes the stable part of $\mathbb{P}\left(\operatorname{Sym}^{3}\left(\mathbb{C}^{2}\right) \otimes \mathbb{C}^{r+1}\right)$. Using this theorem, we could calculate the cohomology ring and the Picard group of the Kontsevich's moduli space of stable maps $\mathcal{M}_{0}\left(\mathbb{P}^{\mathrm{r}}, 3\right)$.

Remark 1.2. Theorem 1.1 holds true for any $r \geq 1$. When $r=1, \pi_{3}$ is the identity map and $\pi_{2}$ cancels out $\pi_{4}$ while $\pi_{1}$ cancels out $\pi_{5}$. Therefore, we have an isomorphism

$$
\mathcal{M}_{0}\left(\mathbb{P}^{1}, 3\right) \cong \mathbb{P}\left(\operatorname{Sym}^{3}\left(\mathbb{C}^{2}\right) \otimes \mathbb{C}^{2}\right) / / \operatorname{SL}(2) .
$$

The fourth compactification is by Simpson's moduli space of stable sheaves. Recall that a coherent sheaf $E$ is pure if any nonzero subsheaf of $E$ has the same dimensional support as E. A pure sheaf $\mathrm{E}$ is called semistable if

$$
\frac{\chi(E(m))}{r(E)} \leq \frac{\chi\left(E^{\prime}(m)\right)}{r\left(E^{\prime}\right)} \quad \text { for } m>>0
$$

for any nontrivial pure quotient sheaf $E^{\prime}$ of the same dimension, where $r(E)$ denotes the leading coefficient of the Hilbert polynomial $\chi(\mathrm{E}(\mathrm{m}))$. We obtain stability if $\leq$ is replaced by $<$. Simpson proved that there is a projective moduli scheme $\operatorname{Simp}^{\mathrm{P}}\left(\mathbb{P}^{\mathrm{r}}\right)$ of semistable sheaves of given Hilbert polynomial P. It is easy to see that semistability coincides with stability when $P(m)=3 m+1$. If $C$ is a smooth rational cubic curve in $\mathbb{P}^{r}$, then the structure sheaf $\mathcal{O}_{C}$ is a stable sheaf. Hence we get an open immersion $\mathbf{X}_{0} \hookrightarrow \mathcal{S i m p}{ }^{3 m+1}\left(\mathbb{P}^{r}\right)$. By taking the closure we obtain a compactifiction $\mathbf{S}$, which we call the Simpson compactification.

In $\S 3$, we compare the Hilbert compactification $\mathbf{H}$ with the Simpson compactification $\mathbf{S}$. For $\mathbf{r}=3$, Freiermuth and Trautmann proved in [3] that $\mathbf{H} \cong \mathbf{S}$. For arbitrary $r$, we prove the following

Proposition 1.3. There is a morphism $\mathbf{H} \rightarrow \mathbf{S}$ which is the blow-up along the smooth locus of stable sheaves with planar support.

When $r=3$, the locus of planar stable sheaves is a divisor and hence we obtain an isomorphism $\mathbf{H} \cong \mathbf{S}$. One direct way to prove this proposition is to construct a family of stable sheaves parameterized by $\mathbf{H}$. The structure sheaves of the closed subschemes parameterized by $\mathbf{H}$ are stable except along the locus of planar cubic curves, which is a divisor. By applying elementary modification, we obtain a family of stable sheaves and thus a morphism from $\mathbf{H}$ to $\mathbf{S}$. Then one can check that this is a blow-down. Another way to prove this is to use a result of Freiermuth and Trautmann ([3]) which we explain in $\S 3$.

In $\S 4$, we compare the Kontsevich compactification $\mathbf{M}$ and the Simpson compactification $\mathbf{S}$. Let $\mathrm{f}: \mathrm{C} \rightarrow \mathbb{P}^{\mathrm{r}}$ be a stable map. Then $\mathrm{f}_{*} \mathcal{O}_{\mathrm{C}}$ is a coherent sheaf on $\mathbb{P}^{r}$. The locus of unstable sheaves turns out to consist of two irreducible components $\Gamma^{1} \cup \Gamma^{2}$, where $\Gamma^{1}$ is the locus of stable maps with linear image (i.e. the image is a line) while $\Gamma^{2}$ is the locus of stable maps with bilinear image (i.e. the image is the union of two lines). To resolve the indeterminacy we first blow up along $\Gamma^{1}$ and apply elementary modification with respect to the destabilizing subsheaves which we define as the first nonzero terms in the Harder-Narasimhan filtrations. Then the locus of unstable sheaves has still two components: one is the proper transform of $\Gamma^{2}$ and the other $\Gamma^{3}$ is a subvariety of the exceptional divisor of the blow-up. We then blow up along $\Gamma^{2}$ and apply the elementary modification with respect to the destabilizing subsheaves. Again we blow up along $\Gamma^{3}$ and apply elementary modification. After these three blow-ups, we obtain a family of stable sheaves and 
hence a morphism to $\mathbf{S}$. Then we study the geometry of the exceptional divisors. It turns out that the exceptional divisor of the second blow-up becomes a weighted projective bundle over a variety and we can contract this divisor. Then the exceptional divisor of the third blow-up becomes a weighted projective bundle and we can contract this divisor. Finally we contract the exceptional divisor of the first blow-up in a similar fashion. In this local analysis, the main technique is the variation of GIT quotients $[19,1]$. Then it is easy to check that the morphism to $\mathbf{S}$ factors through the blow-downs and the induced map is bijective. By the generalized Riemann existence theorem [8, p442], we deduce that all morphisms are algebraic and the blown-down spaces are algebraic. So we obtain the following

Theorem 1.4. $\mathbf{S}$ is obtained from $\mathbf{M}$ by blowing up along $\Gamma^{1}, \Gamma_{1}^{2}, \Gamma_{2}^{3}$ and then blowing down along $\Gamma_{3}^{2}, \Gamma_{4}^{3}, \Gamma_{5}^{1}$, where $\Gamma_{j}^{i}$ denote the proper transform of $\Gamma^{i}$ at the $\mathrm{j}$ th stage.

The following diagram summarizes the results of this paper and [11].

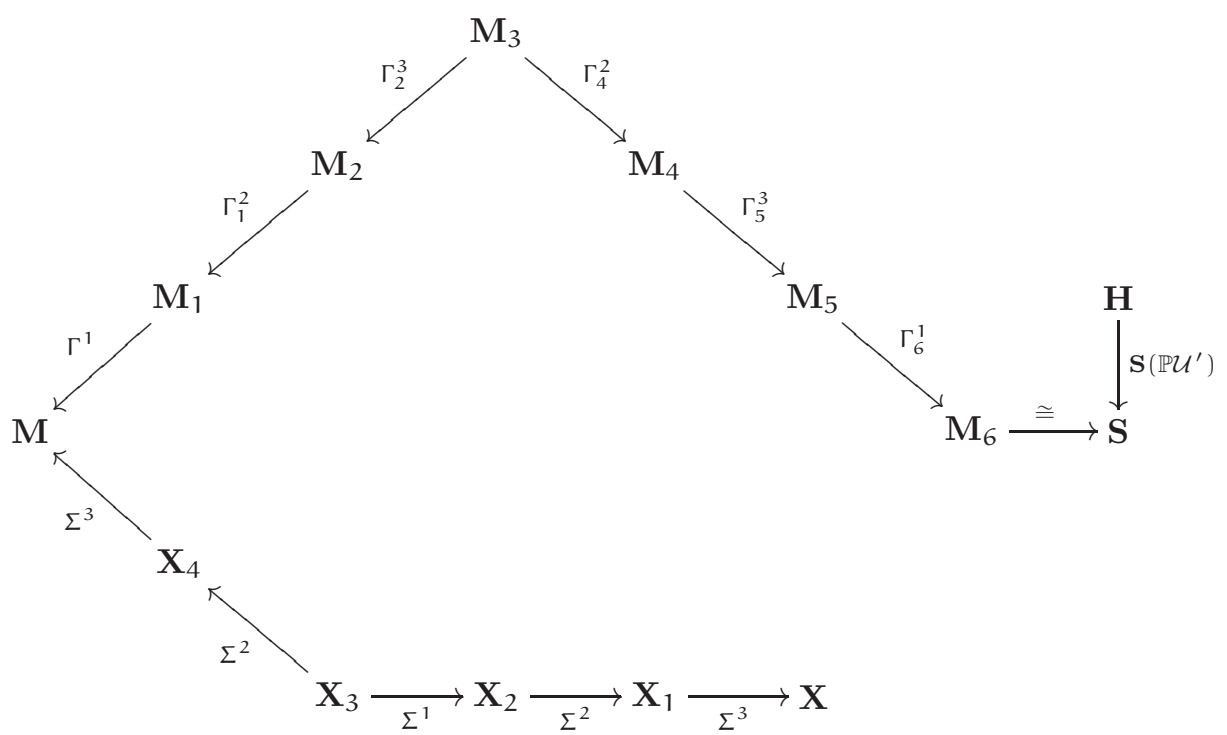

All the arrows are blow-ups and the blow-up centers are indicated above the arrows.

In $\S 5$, we calculate the Betti numbers of $\mathbf{S}$ by using Theorem 1.4. When $r=3$, we get exactly the same numbers, calculated by Ellingsrud, Piene and Stromme [2].

There are other interesting compactifications, such as the Chow compactification, the variety of nets of quadrics [2], the Vainsencher-Xavier compactification [20] and the variety of triples [16]. When $r=3$, the variety of nets of quadrics was shown to be a blow-down of $\mathbf{H}$ but the relationships for other compactifications are not known. We hope to compare them with $\mathbf{X}, \mathbf{H}, \mathbf{M}, \mathbf{S}$ in the future.

\section{Compactifications of the space of Rational Cubics}

In this section, we recall several well-known compactifications of the space of curves: compactifications by the Hilbert scheme, Kontsevich's moduli space of stable maps, Simpson's moduli space of stable sheaves and the space of quasi-maps. Our goal is to compare these compactifications. We fix a positive integer $r$. 
2.1. Compactification by quasi-maps. This is perhaps the easiest to describe. A smooth rational curve of degree 3 in projective space $\mathbb{P}^{r}$, is given by an $(r+1)$ tuple $\left(f_{0}: f_{1}: \cdots: f_{r}\right)$ of degree 3 homogeneous polynomials in two variables $z_{0}, z_{1}$, the homogeneous coordinates of $\mathbb{P}^{1}$. Upon fixing the basis $z_{0}^{3}, z_{0}^{2} z_{1}, z_{0} z_{1}^{2}, z_{1}^{3}$ of the space of degree 3 homogeneous polynomials, the curve is determined by a $4 \times(r+1)$ matrix of coefficients. Whenever this matrix has maximal rank, we get a smooth rational cubic in $\mathbb{P}^{r}$ and two such matrices determine the same curve if and only if they are in the same orbit under the action of $A u t\left(\mathbb{P}^{1}\right)=\operatorname{PGL}(2)$. Hence the space of rational cubics in $\mathbb{P}^{r}$ can be described as

$$
\mathbf{X}_{0}:=\mathbb{P}\left(\operatorname{Sym}^{3}\left(\mathbb{C}^{2}\right) \otimes \mathbb{C}^{\mathbf{r}+1}\right)_{4} / \operatorname{PGL}(2)
$$

where the subscript 4 denotes the open subset of rank 4 matrices. Thus, Geometric Invariant Theory (GIT) provides us with a natural compactification

$$
\mathbf{X}:=\mathbb{P}\left(\operatorname{Sym}^{3}\left(\mathbb{C}^{2}\right) \otimes \mathbb{C}^{\mathrm{r}+1}\right) / / \operatorname{PGL}(2)
$$

which is often called, the quasi-map space. Thanks to the Atiyah-Bott-Kirwan theory, we can easily calculate the cohomology ring/topological K-group/Chow ring of this compactification $[12,11]$. However, the geometric meaning of its boundary points is not clear.

2.2. Kontsevich's moduli space of stable maps. In early 1990's, Kontsevich introduced the notion of stable maps and a compactification of the space of smooth curves, called the moduli space of stable maps. See [5] for an introduction.

By definition, a stable map to $\mathbb{P}^{r}$ is a morphism $f: C \rightarrow \mathbb{P}^{r}$ of a connected reduced curve $C$ which may have only nodal singularities $(x y=0)$, such that the automorphism group of $f$ is finite. If we fix the arithmetic genus $g$ of $C$ and the homology class $d=f_{*}[C]$, then we obtain a projective moduli space $\mathcal{M}_{g}\left(\mathbb{P}^{r}, d\right)$, which parameterizes isomorphism classes of stable maps. When $g=0, \mathcal{M}_{0}\left(\mathbb{P}^{r}, d\right)$ is an irreducible normal projective variety with only finite quotient singularities.

Our concern in this paper is

$$
\mathbf{M}:=\mathcal{M}_{0}\left(\mathbb{P}^{r}, 3\right)
$$

which is obviously a compactification of the space $\mathbf{X}_{0}$ of smooth rational cubics. As the first step of our project of comparing various compactifications of $\mathbf{X}_{0}$, we proved in [11], that $\mathbf{M}$ is obtained from $\mathbf{X}$ by three blow-ups followed by two blow-downs. The blow-up/-down centers were all described in terms of moduli spaces of stable maps of degrees 1 and 2. These results enabled us to do various cohomological calculations on $\mathbf{M}$. In this paper, we compare $\mathbf{M}$ with other moduli theoretic compactifications described below. We will call $\mathbf{M}$ the Kontsevich compactification. We will write $\mathbf{M}\left(\mathbb{P}^{r}\right)$ if it is necessary to emphasize the target space $\mathbb{P}^{r}$.

2.3. Hilbert scheme and Chow scheme. Classical approaches to compactification of the space of smooth curves are to use either the Hilbert scheme or the Chow scheme. See $[14,7]$ for an introduction.

The Hilbert scheme $\mathcal{H i l b}^{\mathrm{P}}\left(\mathbb{P}^{\mathrm{r}}\right)$ is the projective moduli space of closed subschemes in $\mathbb{P}^{r}$ whose Hilbert polynomial is $\mathrm{P}$. The space of smooth rational cubics in $\mathbb{P}^{r}$ form an open subset of $\mathcal{H} \mathrm{ilb}^{3 \mathrm{~m}+1}\left(\mathbb{P}^{\mathrm{r}}\right)$ and we call its closure $\mathbf{H}$, the Hilbert compactification. When it is necessary to emphasize the target space $\mathbb{P}^{r}$, we will write $\mathbf{H}\left(\mathbb{P}^{r}\right)$ instead of $\mathbf{H}$. 
The Chow scheme $\mathcal{C}$ how $w^{1,3}\left(\mathbb{P}^{r}\right)$ of one dimensional cycles of degree three is a projective scheme which contains $\mathbf{X}_{0}$ as an open subset. We denote by $\mathbf{C}$ the closure of $\mathbf{X}_{0}$ in the Chow scheme and call it the Chow compacticiation. $\mathbf{H}$ is smooth and we have a natural morphism $\mathrm{HC}: \mathbf{H} \rightarrow \mathbf{C}$ forgetting the thickening structure of multiple components. Furthermore, there is a natural morphism $\mathbf{M} \rightarrow \mathbf{C}$ which forgets the ramification points of multiple components.

2.4. Simpson's moduli space of stable sheaves. Let $E$ be a coherent sheaf on $\mathbb{P}^{r}$. We say $E$ is pure if any nonzero subsheaf of $E$ has the same dimensional support as $\mathrm{E}$. A pure sheaf $\mathrm{E}$ is called semistable if

$$
\frac{\chi(E(m))}{r(E)} \leq \frac{\chi\left(E^{\prime}(m)\right)}{r\left(E^{\prime}\right)} \quad \text { for } m>>0
$$

for any nontrivial pure quotient sheaf $E^{\prime}$ of the same dimension, where $r(E)$ denotes the leading coefficient of the Hilbert polynomial $\chi(E(m))$. We obtain stability if $\leq$ is replaced by $<$.

In [18], Simpson proved by GIT that there is a projective moduli scheme $\mathcal{S i m p}^{\mathrm{P}}\left(\mathbb{P}^{\mathrm{r}}\right)$ of equivalence classes of semistable sheaves on $\mathbb{P}^{r}$ whose Hilbert polynomial is $P$. The following easy lemma explains why $\mathcal{S i m p}^{3 m+1}\left(\mathbb{P}^{r}\right)$ gives us a compactification of $\mathbf{X}_{0}$.

Lemma 2.1. [3, Lemma 1] If C is a Cohen-Macaulay curve of degree 3 in $\mathbb{P}^{r}$, its structure sheaf $\mathcal{O}_{\mathrm{C}}$ is a stable sheaf.

Proof. Since $\mathcal{O}_{\mathrm{C}}$ is a quotient of $\mathcal{O}_{\mathbb{P}^{\mathrm{r}}}$, any nontrivial pure quotient of $\mathcal{O}_{\mathrm{C}}$ is the structure sheaf $\mathcal{O}_{C^{\prime}}$ of a closed subcurve $C^{\prime}$ of $C$. As the degree of $C^{\prime}$ is at most two, the support of $C^{\prime}$ is a line or a conic or a pair of lines. In any case,

$$
m+\frac{1}{3}=\frac{\chi\left(\mathcal{O}_{\mathrm{C}}(\mathrm{m})\right)}{r\left(\mathcal{O}_{\mathrm{C}}\right)}<\frac{\chi\left(\mathcal{O}_{\mathrm{C}^{\prime}}(\mathrm{m})\right)}{\mathrm{r}\left(\mathcal{O}_{\mathrm{C}^{\prime}}\right)}=\mathrm{m}+1, \text { or } \mathrm{m}+\frac{1}{2} \text {. }
$$

By an easy calculation, we have an identification of tangent spaces

$$
\mathrm{T}_{\mathcal{O}_{\mathrm{C}}} \mathcal{S i m p}^{3 \mathrm{~m}+1}\left(\mathbb{P}^{\mathrm{r}}\right)=\mathrm{Ext}_{\mathbb{P}^{\mathrm{r}}}^{1}\left(\mathcal{O}_{\mathrm{C}}, \mathcal{O}_{\mathrm{C}}\right) \cong \mathrm{H}^{\mathrm{O}}\left(\mathrm{C}, \mathrm{N}_{\mathrm{C} / \mathbb{P}^{\mathrm{r}}}\right)=\mathrm{T}_{[\mathrm{C}]} \mathbf{X}_{\mathrm{O}}
$$

for a smooth rational cubic $\mathrm{C} \in \mathbf{X}_{0}$. Hence, via the map $\mathrm{C} \mapsto \mathcal{O}_{\mathrm{C}}, \mathbf{X}_{0}$ is isomorphic to an open subset of $\mathcal{S i m p}^{3 \mathrm{~m}+1}\left(\mathbb{P}^{\mathrm{r}}\right)$. Let $\mathbf{S}$ be the irreducible component of $\operatorname{Simp}{ }^{3 m+1}\left(\mathbb{P}^{r}\right)$ which contains $\mathbf{X}_{0}$. We call this the Simpson compactification. When it is necessary to emphasize the target space $\mathbb{P}^{r}$, we will write $\mathbf{S}\left(\mathbb{P}^{r}\right)$ instead of $\mathbf{S}$.

In $\S 3$, we compare $\mathbf{H}$ and $\mathbf{S}$ and in $\S 4$, we compare $\mathbf{M}$ and $\mathbf{S}$. Their relationships will be described in terms of explicit blow-ups and -down.

\section{From Hilbert to Simpson}

In this section, we prove that the Hilbert compactification $\mathbf{H}$ is the blow-up of the Simpson compactification $\mathbf{S}$ along a smooth subvariety. One way to prove this is as follows: the universal family $\mathcal{Z} \subset \mathbb{P}^{r} \times \mathbf{H}$ defines a family of sheaves $\mathcal{O}_{\mathcal{Z}}$ on $\mathbb{P}^{r} \times \mathbf{H}$ which is flat over $\mathbf{H}$. The locus of unstable sheaves is a smooth divisor $\Delta$ and the destabilizing subsheaves are zero dimensional. We then apply the elementary modification

$$
\mathcal{F}:=\operatorname{ker}\left(\left.\mathcal{O}_{\mathcal{Z}} \rightarrow \mathcal{O}_{\mathcal{Z}}\right|_{\Delta} \rightarrow A\right)
$$


where $A$ is the destabilizing quotient. Then one can check that $\mathcal{F}$ is a flat family of stable sheaves on $\mathbb{P}^{r} \times \mathbf{H}$ and hence we obtain a morphism $\mathbf{H} \rightarrow \mathbf{S}$. By further analyzing fibers, one can show that this is a blow-up map or a divisorial contraction of $\Delta$ as we do below.

Instead of the above method of using elementary modification, we take a shorter path of using the results of $[17,3]$.

Theorem 3.1. [17] $\mathcal{H i l b}^{3 \mathrm{~m}+1}\left(\mathbb{P}^{3}\right)$ has only two irreducible components. They are smooth and intersect transversely. One of them is $\mathbf{H}\left(\mathbb{P}^{3}\right)$ and the other is a 15 dimensional variety parameterizing planar cubics coupled with points. Their intersection is a divisor $\Delta\left(\mathbb{P}^{3}\right)$ of $\mathbf{H}\left(\mathbb{P}^{3}\right)$.

Theorem 3.2. [3]

(1) $\operatorname{Simp}^{3 m+1}\left(\mathbb{P}^{3}\right)$ is the fine moduli space of stable sheaves, i.e. semistable sheaves are stable.

(2) $\mathcal{S i m p}^{3 m+1}\left(\mathbb{P}^{3}\right)$ has two irreducible components which intersect transversely along $\Delta\left(\mathbb{P}^{3}\right)$. One is $\mathbf{S}\left(\mathbb{P}^{3}\right)$ and the other is a 13 dimensional variety which parameterizes planar cubics together with marked points.

(3) $\mathbf{S}\left(\mathbb{P}^{3}\right)$ is isomorphic to $\mathbf{H}\left(\mathbb{P}^{3}\right)$.

From these theorems, we obtain the following.

Proposition 3.3. $\quad$ (1) $\mathbf{H}\left(\mathbb{P}^{\mathrm{r}}\right)$ is isomorphic to $\mathbf{H}(\mathbb{P U})$ which is a component of the relative Hilbert scheme for the bundle $\mathbb{P U} \rightarrow \mathrm{Gr}(4, r+1)$ of $\mathbb{P}^{3}$ 's, where $\mathcal{U}$ is the universal rank 4 vector bundle on the Grassmannian $\mathrm{Gr}(4, \mathrm{r}+1)$.

(2) $\mathbf{H}\left(\mathbb{P}^{r}\right)$ is the blow-up of $\mathbf{S}\left(\mathbb{P}^{r}\right)$ along the smooth locus of planar stable sheaves. In particular, $\mathbf{S}\left(\mathbb{P}^{r}\right)$ is nonsingular.

Proof. (1) The relative Hilbert scheme $\mathcal{H i l b}^{3 m+1}(\mathbb{P U})$ over $\mathrm{Gr}(4, \mathrm{r}+1)$ has fibers $\mathcal{H i l b}{ }^{3 m+1}\left(\mathbb{P}^{3}\right)$. By Theorem 3.1, we obtain an irreducible component $\mathbf{H}(\mathbb{P} \mathcal{U})$ whose fibers are $\mathbf{H}\left(\mathbb{P}^{3}\right)$. We then have a natural morphism

$$
\phi: \mathbf{H}(\mathbb{P U}) \rightarrow \mathbf{H}\left(\mathbb{P}^{r}\right)
$$

sending $Z \subset \mathbb{P U}$ to $Z \subset \mathbb{P}^{r}$ via the inclusion $\mathbb{P U} \hookrightarrow \mathbb{P}^{r}$ for any $\mathrm{U} \in \mathrm{Gr}(4, \mathrm{r}+1)$. By Theorem 3.1, $\mathbf{H}(\mathbb{P U})$ is smooth and proper. Since both $\mathbf{H}(\mathbb{P U})$ and $\mathbf{H}\left(\mathbb{P}^{r}\right)$ are compactifications of the space of smooth rational cubics in $\mathbb{P}^{r}, \phi$ is birational.

In [7], all possible types of elements in $\mathbf{H}\left(\mathbb{P}^{3}\right)$ are described. Looking at the list, one immediately sees that $\phi$ is injective because the image $\phi(y)$ of every point $y \in \mathbf{H}(\mathbb{P U})$ determines a unique $\mathbb{P}^{3}$. This certainly implies that $\phi$ is bijective. By Zariski's main theorem, we deduce that $\phi$ is an isomorphism.

(2) Similarly as above, we have a smooth family $\mathbf{S}(\mathbb{P U}) \rightarrow \mathrm{Gr}(4, r+1)$ whose fibers are $\mathbf{S}(\mathbb{P U})$ for $U \in G r(4, r+1)$. By Theorem 3.2, $\mathbf{H}(\mathbb{P U}) \cong \mathbf{S}(\mathbb{P U})$ and thus it suffices to study the relationship between $\mathbf{S}(\mathbb{P U})$ and $\mathbf{S}\left(\mathbb{P}^{r}\right)$. The inclusion $\imath: \mathbb{P U} \hookrightarrow \mathbb{P}^{r}$ for $\mathrm{U} \in \mathrm{Gr}(4, \mathrm{r}+1)$ induces a morphism

$$
\psi: \mathbf{S}(\mathbb{P U}) \rightarrow \mathbf{S}\left(\mathbb{P}^{\mathrm{r}}\right)
$$

by sending $F$ to $\imath_{*} F$. This is an isomorphism on the complement of the divisor $\Delta$ of planar sheaves. To describe this divisor, let $\mathbf{S}\left(\mathbb{P}^{2}\right) \subset \mathcal{S i m p}{ }^{3 m+1}\left(\mathbb{P}^{2}\right)$ be the locus of sheaves $\mathrm{F}$ each of which is a nontrivial extension

$$
0 \rightarrow \mathcal{O}_{C} \rightarrow F \rightarrow \mathbb{C}_{p} \rightarrow 0
$$


for a singular planar cubic $C$ and a singular point $p \in C$. See [3, Lemma 2]. Then $\Delta$ is a $\mathbf{S}\left(\mathbb{P}^{2}\right)$-bundle on $\mathbb{P U}^{*}$ over $\operatorname{Gr}(4, r+1)$. By Theorem 3.2, $\Delta$ is smooth when $r=3$ and hence $\mathbf{S}\left(\mathbb{P}^{2}\right)$ is nonsingular.

Using the isomorphism $\mathbb{P} \mathcal{U}^{*} \cong \mathbb{P}\left(\mathbb{C}^{r+1} / \mathcal{U}^{\prime}\right)$ where $\mathcal{U}^{\prime}$ is the universal rank 3 vector bundle on $\operatorname{Gr}(3, r+1)$, we see that $\Delta$ is isomorphic to $\mathbf{S}\left(\mathbb{P} \mathcal{U}^{\prime}\right) \times \operatorname{Gr}(3, r+1)$ $\mathbb{P}\left(\mathbb{C}^{r+1} / \mathcal{U}^{\prime}\right)$ over $\operatorname{Gr}(3, r+1)$. Obviously, the sheaf $F$ is independent of the choice of $\mathbb{P}^{3}$ containing the support and hence $\psi$ is constant on the fibers $\mathbb{P}^{r-3}$ of $\mathbb{P}\left(\mathbb{C}^{r+1} / \mathcal{U}^{\prime}\right) \rightarrow$ $\operatorname{Gr}(3, r+1)$. To deduce that $\psi$ is the blow-up map along $\mathbf{S}\left(\mathbb{P} \mathcal{U}^{\prime}\right)$ and hence $\mathbf{S}\left(\mathbb{P}^{r}\right)$ is smooth, we only need to show that the normal bundle of $\Delta$ restricted to a fiber of $\mathbb{P}\left(\mathbb{C}^{r+1} / \mathcal{U}^{\prime}\right) \rightarrow \operatorname{Gr}(3, r+1)$ is $\mathcal{O}_{\mathbb{P} r-3}(-1)$. But this can be easily checked. For instance, suppose $\mathrm{C}$ is the planar curve given by a map $\mathbb{P}^{1} \rightarrow \mathbb{P}^{r}$

$$
\left(z_{0}^{3}: z_{0}^{2} z_{1}+z_{0} z_{1}^{2}: z_{1}^{3}: 0: \cdots: 0\right)
$$

and consider the family of smooth rational curves given by

$\left(z_{0}^{3}: z_{0}^{2} z_{1}+z_{0} z_{1}^{2}: z_{1}^{3}: a_{1} z_{0} z_{1}^{2}: a_{2} z_{0} z_{1}^{2}: \cdots: a_{r-2} z_{0} z_{1}^{2}\right), \quad\left(a_{1}, \cdots, a_{r-2}\right) \in \mathbb{C}^{r-2}-\{0\}$. This gives us a morphism $\mathbb{C}^{r-2}-\{0\} \rightarrow \mathbf{S}\left(\mathbb{P}^{r}\right)$ which has a unique extension $\mathbb{C}^{r-2} \rightarrow$ $\mathbf{S}\left(\mathbb{P}^{r}\right)$, whose central image $F$ fits into a nonsplit exact sequence

$$
0 \rightarrow \mathcal{O}_{\mathrm{C}} \rightarrow \mathrm{F} \rightarrow \mathbb{C}_{\mathrm{p}} \rightarrow 0
$$

where $p$ is the unique nodal point. This extension is of course obtained by taking the direct image $f_{*} \mathcal{O}_{\mathbb{P} 1}$ for each $f: \mathbb{P}^{1} \rightarrow \mathbb{P}^{r}$ in the family parameterized by $\mathbb{C}^{r-2}$. On the other hand, we have a morphism $\mathbb{C}^{r-2}-\{0\} \rightarrow \mathbf{S}(\mathbb{P} \mathcal{U})$ which extends to $\mathcal{O}_{\mathbb{P}^{r-3}}(-1) \rightarrow \mathbf{S}(\mathbb{P U})$ by taking direct image after choosing a $\mathbb{P}^{3}$ containing the plane of $\mathrm{C}$. This means the normal bundle restricts to $\mathcal{O}(-1)$ as desired. Since $\Delta$ is flat over $\mathbf{S}\left(\mathbb{P} \mathcal{U}^{\prime}\right)$, this holds for every fiber.

\section{From Kontsevich to Simpson}

In this section, we compare the Kontsevich compactification $\mathbf{M}$ and the Simpson compactification $\mathbf{S}$. We will prove that the birational map $\mathbf{M} \rightarrow \mathbf{S}$ is the composition of three blow-ups and three blow-downs, whose centers will be described explicitly below.

Let us consider any family of stable maps of degree 3 parameterized by a reduced scheme Z

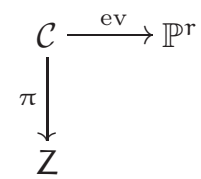

where $\pi$ is a family of connected curves of arithmetic genus 0 , with at worst nodal singularities. Let $\mathcal{E}_{0}$ be the direct image of $\mathcal{O}_{\mathcal{C}}$ by $(\mathrm{ev}, \pi): \mathcal{C} \rightarrow \mathbb{P}^{\mathrm{r}} \times \mathrm{Z}$. Then $\mathcal{E}_{0}$ is a family of coherent sheaves on $\mathbb{P}^{r}$, flat over $\mathbf{Z}$ because the Hilbert polynomial is constantly $3 m+1$. By Lemma 2.1 , the restriction of $\mathcal{E}_{0}$ to $\mathbb{P}^{r} \times Z_{0}$ where $Z_{0}$ is the locus of smooth curves, is a family of stable sheaves on $\mathbb{P}^{r}$. Hence we obtain a birational map

$$
\Phi: \mathbf{M} \rightarrow \mathbf{S} .
$$

How do we eliminate the locus of indeterminacy? First we find the locus of indeterminacy. Next we find a suitable sequence of blow-ups and apply elementary modification to construct a family of stable sheaves. Thus we get a morphism to 
S. Finally we study the local geometry of the exceptional divisors and show that we can contract the divisors. After these blow-downs, we obtain an isomorphism of the resulting model of $\mathbf{M}$ with $\mathbf{S}$.

4.1. Locus of indeterminacy. To find the locus of indeterminacy of $\Phi$, we need the following generalization of Lemma 2.1.

Lemma 4.1. If $\mathrm{f}: \mathrm{C} \rightarrow \mathbb{P}^{\mathrm{r}}$ is a stable map in $\mathbf{M}$ with no multiple components (i.e. no component of $\mathrm{f}(\mathrm{C})$ is a multiply covered by $\mathrm{f})$, then $\mathrm{f}_{*} \mathcal{O}_{\mathrm{C}}$ is a stable sheaf.

Proof. If $\mathrm{f}$ is non-planar, $\mathrm{C}^{\prime}=\mathrm{f}(\mathrm{C})$ is a Cohen-Macaulay curve and $\mathrm{f}_{*} \mathcal{O}_{\mathrm{C}}=\mathcal{O}_{\mathrm{C}^{\prime}}$. By Lemma $2.1, f_{*} \mathcal{O}_{C}$ is stable. Suppose now $f$ is planar. By our assumption, $C^{\prime}$ is a singular cubic and $f_{*} \mathcal{O}_{C}$ fits into an exact sequence

$$
0 \rightarrow \mathcal{O}_{\mathrm{C}^{\prime}} \rightarrow \mathrm{f}_{*} \mathcal{O}_{\mathrm{C}} \rightarrow \mathcal{O}_{\mathrm{p}} \rightarrow 0
$$

for some point $p \in C^{\prime}$ because the Hilbert polynomial of $\mathcal{O}_{C^{\prime}}$ is $3 \mathrm{~m}$. The first map comes from the adjunction $\mathcal{O}_{\mathbb{P}} \rightarrow f_{*} f^{*} \mathcal{O}_{\mathbb{P}^{r}}=f_{*} \mathcal{O}_{\mathrm{C}}$. Since $\operatorname{Hom}\left(\mathcal{O}_{p}, f_{*} \mathcal{O}_{\mathrm{C}}\right)=$ $\operatorname{Hom}\left(f^{*} \mathcal{O}_{p}, \mathcal{O}_{C}\right)=0, f_{*} \mathcal{O}_{C}$ is a nonsplit extension. Because $C^{\prime}$ is Cohen-Macaulay, $\mathcal{O}_{C^{\prime}}$ is stable. It is easy to see now that $f_{*} \mathcal{O}_{C}$ is stable.

Consequently, the locus of unstable sheaves in the family $\mathcal{E}_{0}$ has two irreducible components: one component $\Gamma^{1}$ consists of stable maps, each of which has a single line as its image, and the other component $\Gamma^{2}$ consists of stable maps, each of which has a pair of lines as its image. Let $\mathrm{Gr}(2, r+1)$ be the Grassmannian of two dimensional subspaces of $\mathbb{C}^{r+1}$ and $\mathcal{U}$ be the universal rank 2 bundle. Let $\mathcal{M}_{0}(\mathbb{P U}, \mathrm{d})$ be the relative moduli space of stable maps of degree $\mathrm{d}$ and arithmetic genus 0 to the fibers of $\mathbb{P U} \rightarrow \operatorname{Gr}(2, r+1)$. The obvious map $\mathcal{M}_{0}(\mathbb{P U}, 3) \rightarrow$ $\operatorname{Gr}(2, r+1)$ is a locally trivial bundle with fiber $\mathcal{M}_{0}\left(\mathbb{P}^{1}, 3\right)$ and we have an inclusion map

$$
\Gamma^{1}:=\mathcal{M}_{0}(\mathbb{P U}, 3) \hookrightarrow \mathcal{M}_{0}\left(\mathbb{P}^{r}, 3\right) .
$$

By gluing operation at the marked points, we see that the component $\Gamma^{2}$ is isomorphic to the fiber product

$$
\mathcal{M}_{0,1}(\mathbb{P U}, 2) \times_{\mathbb{P} r} \mathbb{P U}
$$

where $\mathcal{M}_{0,1}(\mathbb{P U}, 2)$ is the moduli space of stable maps of genus 0 and degree 2 with one marked point to the fibers of $\mathbb{P} \mathcal{U} \rightarrow \operatorname{Gr}(2, r+1)$. Note here that $\mathcal{M}_{0,1}\left(\mathbb{P}^{r}, 1\right)=$ $\mathbb{P U} \cong \mathbb{P}_{\mathbb{P} r}$ and the marked points give us morphisms to $\mathbb{P}^{r}$ in the fiber product. For further analysis however, we need a description of $\Gamma^{1}$ and $\Gamma^{2}$ via GIT.

4.2. Indeterminacy via GIT. In $[11, \S 5]$, we proved that

$$
\mathrm{M} \cong \mathrm{Q}_{0} / \mathrm{SL}(2)
$$

for a smooth quasi-projective variety $\mathbf{Q}_{0}=\mathbf{P}_{5}$. Let us briefly recall the construction.

We start with the stable part $\mathbf{P}_{0}$ of the projective space $\mathbb{P}\left(\operatorname{Sym}^{3}\left(\mathbb{C}^{2}\right) \otimes \mathbb{C}^{r+1}\right)$ with respect to the action of $\operatorname{SL}(2)$ on $\operatorname{Sym}^{3}\left(\mathbb{C}^{2}\right)$. An element in $\mathbf{P}_{0}$ can be thought of as an $(r+1)$-tuple $\left(f_{0}: \cdots: f_{r}\right)$ of homogeneous polynomials in $z_{0}, z_{1}$ of degree 3. When there are no common zeroes of these $(r+1)$ polynomials, we get a stable map. Let $\pi_{1}: \mathbf{P}_{1} \rightarrow \mathbf{P}_{0}$ be the blow-up along the locus $\Sigma^{3}$ of three common zeroes. Next let $\pi_{2}: \mathbf{P}_{2} \rightarrow \mathbf{P}_{1}$ be the blow-up along the proper transform of the locus $\bar{\Sigma}^{2}$ 
of at least two common zeros. Let $\pi_{3}: \mathbf{P}_{3} \rightarrow \mathbf{P}_{2}$ be the blow-up along the proper transform of the locus $\bar{\Sigma}^{1}$ of at least one common zero. After these three blow-ups and elementary modifications along the exceptional divisors, we obtain a family of stable maps

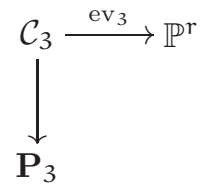

parameterized by $\mathbf{P}_{3}$ and thus a morphism $\mathbf{P}_{3} \rightarrow \mathbf{M}$.

The exceptional divisor of the second blow-up becomes a $\mathbb{P}^{1}$-bundle and the normal bundle restricted to each fiber $\mathbb{P}^{1}$ is $\mathcal{O}(-1)$. Hence we can contract this divisor to obtain $\pi_{4}: \mathbf{P}_{3} \rightarrow \mathbf{P}_{4}$. Then the exceptional divisor of the first blow-up becomes a $\mathbb{P}^{2}$-bundle and the normal bundle restricted to each fiber $\mathbb{P}^{2}$ is $\mathcal{O}(-1)$. Hence we can contract this divisor to obtain $\pi_{5}: \mathbf{P}_{4} \rightarrow \mathbf{P}_{5}$. The morphism $\mathbf{P}_{3} \rightarrow \mathbf{M}$ factors through the two blow-downs and the induced map $\mathbf{P}_{5} \rightarrow \mathbf{M}$ is SL(2)invariant. Therefore, we obtain a morphism $\mathbf{P}_{5} / \mathrm{SL}(2) \rightarrow \mathbf{M}$ which turns out to be a bijection and hence an isomorphism. Thus we obtain the isomorphism (4.2) with $\mathrm{Q}_{0}:=\mathbf{P}_{5}$. Furthermore, by the same argument, $\mathcal{C}_{3}$ can be blown down twice and ev3 factors through

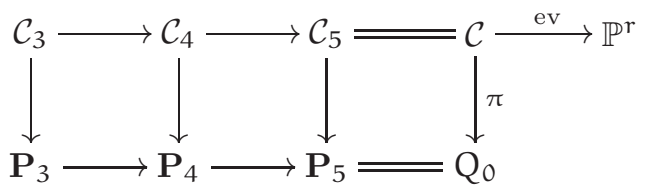

Thus we have a natural morphism

$$
\varphi_{\mathbf{f}}: \mathcal{O}_{\mathbb{P}^{r} \times Q_{0}} \rightarrow \mathbf{f}_{*} \mathcal{O}_{\mathcal{C}}=: \mathcal{E}_{0}
$$

where $\mathbf{f}=(\mathrm{ev}, \boldsymbol{\pi})$.

For a description of $\Gamma^{1}$ as the quotient of a smooth variety, let us consider

$$
\Theta^{1}:=\mathbb{P}\left(\operatorname{Sym}^{3}\left(\mathbb{C}^{2}\right) \otimes \mathbb{C}^{2}\right)^{s} \times_{P G L(2)} \mathbb{P}\left(\mathbb{C}^{2} \otimes \mathbb{C}^{r+1}\right)^{s}
$$

which is a $\mathbb{P}\left(\operatorname{Sym}^{3}\left(\mathbb{C}^{2}\right) \otimes \mathbb{C}^{2}\right)^{\text {s}}$-bundle over $\operatorname{Gr}(2, r+1)$. The subscript PGL(2) denotes the quotient by the diagonal action of PGL(2). Note that the stable part $\mathbb{P}\left(\mathrm{Sym}^{3}\left(\mathbb{C}^{2}\right) \otimes \mathbb{C}^{2}\right)^{s}$ is the stable part with respect to the diagonal action on $\mathbb{P} \operatorname{Sym}^{3}\left(\mathbb{C}^{2}\right)^{\oplus 2}$ while the stable part $\mathbb{P}\left(\mathbb{C}^{2} \otimes \mathbb{C}^{r+1}\right)^{s}$ is with respect to the diagonal action on $\mathbb{P}\left(\left(\mathbb{C}^{2}\right)^{\oplus \mathrm{r}+1}\right)$. We have $\Theta^{1} / \mathrm{SL}(2) \cong \mathcal{M}_{0}(\mathbb{P U}, 3)$ because by [11],

$$
\mathbb{P}\left(\operatorname{Sym}^{3}\left(\mathbb{C}^{2}\right) \otimes \mathbb{C}^{2}\right)^{s} / \operatorname{SL}(2) \cong \mathcal{M}_{0}\left(\mathbb{P}^{1}, 3\right) \text {. }
$$

Further, the obvious composition map

$$
\mathbb{P} \operatorname{Hom}\left(\operatorname{Sym}^{3}\left(\mathbb{C}^{2}\right), \mathbb{C}^{2}\right) \times \mathbb{P} \operatorname{Hom}\left(\mathbb{C}^{2}, \mathbb{C}^{r+1}\right) \longrightarrow \mathbb{P} \operatorname{Hom}\left(\operatorname{Sym}^{3}\left(\mathbb{C}^{2}\right), \mathbb{C}^{r+1}\right)
$$

induces a morphism $\Theta^{1} \rightarrow \mathbf{P}_{0}$. It is straightforward to keep track of the blow-ups and -downs of $\mathbf{P}_{0}$ and see that we obtain an injective morphism

$$
\Theta^{1} \hookrightarrow Q_{0}
$$

whose quotient gives us the embedding $\Gamma^{1} \hookrightarrow \mathbf{M}$. 
Now we provide a description of the other component of the indeterminacy locus via GIT. The set of homogeneous polynomials of degree 1, up to constant multiple, is $\mathbb{P}^{1}$. Multiplication of polynomials and composition (4.4) give us

$$
\left[\mathbb{P}^{1} \times \mathbb{P}\left(\operatorname{Sym}^{2}\left(\mathbb{C}^{2}\right) \otimes \mathbb{C}^{2}\right)\right]^{s} \times{ }_{\mathrm{PGL}(2)} \mathbb{P}\left(\mathbb{C}^{2} \otimes \mathbb{C}^{\mathrm{r}+1}\right)^{\mathrm{s}} \longrightarrow \mathbb{P}\left(\operatorname{Sym}^{3}\left(\mathbb{C}^{2}\right) \otimes \mathbb{C}^{\mathrm{r}+1}\right)^{s}=\mathbf{P}_{0}
$$

where the stability of the first factor is with respect to the diagonal action on $\mathcal{O}(1,1)$ of $\mathbb{P}\left(\mathbb{C}^{2}\right) \times \mathbb{P} \operatorname{Sym}^{2}\left(\mathbb{C}^{2}\right)^{\oplus 2}$ while the stability on the second factor is with respect to the diagonal $\operatorname{SL}(2)$-action on $\mathbb{P}\left(\left(\mathbb{C}^{2}\right)^{\oplus r+1}\right)$. It is also a straightforward exercise to keep track of this morphism through the blow-ups and -downs of $\mathbf{P}_{0}$. The first blow-up $\pi_{1}$ corresponds to the blow-up along

$$
\left(\mathbb{P}^{1} \times\left(\mathbb{P}\left(\operatorname{Sym}^{2} \mathbb{C}^{2}\right) \times \mathbb{P}^{1}\right)\right)^{s} \times \text { PGL(2) } \mathbb{P}\left(\mathbb{C}^{2} \otimes \mathbb{C}^{\mathrm{r}+1}\right)^{s} .
$$

The second blow-up $\pi_{2}$ is the identity map because the blow-up center is a smooth divisor. Then as in the proof of [11, Lemma 5.4], we obtain an equivariant embedding

$$
\left[\mathbb{P}^{1} \times \mathrm{bl}_{\mathbb{P}^{2} \times \mathbb{P}^{1}} \mathbb{P}\left(\operatorname{Sym}^{2}\left(\mathbb{C}^{2}\right) \otimes \mathbb{C}^{2}\right)\right]^{\mathrm{s}} \times \mathrm{PGL}(2) \mathbb{P}\left(\mathbb{C}^{2} \otimes \mathbb{C}^{\mathrm{r}+1}\right)^{\mathrm{s}} \hookrightarrow \mathbf{P}_{2} .
$$

Since the image of (4.7) is contained in the blow-up center, the third blow-up $\pi_{3}$ gives us a $\mathbb{P}^{r-1}$-bundle over the image of (4.7). By the construction of stable maps $[11, \S 5]$, this bundle parameterizes all possible stable maps whose images are two lines. The first blow-down doesn't make any change while the second blow-down contracts the exceptional divisor of the first blow-up. Therefore, we obtain a $\mathbb{P}^{r-1}$ bundle over

$$
\left[\mathbb{P}^{1} \times \mathbb{P}\left(\operatorname{Sym}^{2}\left(\mathbb{C}^{2}\right) \otimes \mathbb{C}^{2}\right)\right]^{s} \times P G L(2) \mathbb{P}\left(\mathbb{C}^{2} \otimes \mathbb{C}^{r+1}\right)^{s}
$$

which we denote by $\Theta^{2}$. Thus, we obtain a smooth subvariety

$$
\Theta^{2} \hookrightarrow Q_{0}
$$

whose quotient gives us the locus of stable maps $\Gamma^{2}$ with bilinear image.

Let us now consider the normal bundle of $\Theta^{1}$. The normal bundle of a fiber $\mathbb{P}\left(\operatorname{Sym}^{3}\left(\mathbb{C}^{2}\right) \otimes \mathbb{C}^{2}\right)^{s}$ of $\Theta^{1}$ over $\operatorname{Gr}(2, r+1)$ in $Q_{0}$ is the pull-back of the normal bundle of $\mathcal{M}_{0}\left(\mathbb{P}^{1}, 3\right)$ in $\mathbf{M}=\mathcal{M}_{0}\left(\mathbb{P}^{r}, 3\right)$ i.e.

$$
\pi_{*} \mathrm{ev}^{*} \mathrm{~N}_{\mathbb{P}^{1} / \mathbb{P}^{\mathrm{r}}}=\left(\pi_{*} \mathrm{ev}^{*} \mathcal{O}_{\mathbb{P}^{1}}(1)\right)^{\oplus \mathrm{r}-1}
$$

where

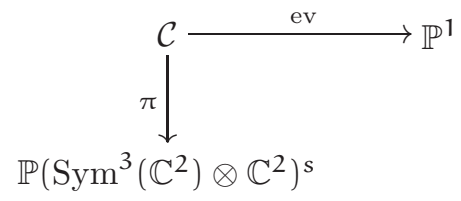

is the family of stable maps to $\mathbb{P}^{1}$.

Lemma 4.2.

$$
\pi_{*} \mathrm{ev}^{*} \mathcal{O}_{\mathbb{P}^{r}}(1) \cong \mathcal{O}_{\mathbb{P}^{7}}^{\oplus 2} \oplus \mathcal{O}_{\mathbb{P}^{7}}(-1)^{\oplus 2} .
$$

Proof. Let $\mathrm{P}_{0}=\mathbb{P}\left(\operatorname{Sym}^{3}\left(\mathbb{C}^{2}\right) \otimes \mathbb{C}^{2}\right)^{s}$. Since the complement of $\mathrm{P}_{0}$ has codimension 3 , the vector bundle $\pi_{*} \mathrm{e} v^{*} \mathcal{O}(1)$ on $\mathrm{P}_{0}$ extends uniquely to a vector bundle $\mathrm{E}$ on $\mathbb{P}^{7}$. By the splitting criterion of Horrocks [15, Theorem 2.3.1], it suffices to prove that

(1) $H^{i}\left(\mathbb{P}^{7}, E(k)\right)=0$ for $i=1,2, \cdots, 6$ and $k \in \mathbb{Z}$. 
(2) The Hilbert polynomial $\mathrm{H}^{0}\left(\mathbb{P}^{7}, \mathrm{E}(\mathrm{k})\right)$ of $\mathrm{E}$ coincides with that of $\mathcal{O}_{\mathbb{P}^{7}}^{\oplus 2} \oplus$ $\mathcal{O}_{\mathbb{P}^{7}}(-1)^{\oplus 2}$.

The first condition tells us that the bundle E splits into a direct sum of line bundles and the second condition shows that the line bundles are two $\mathcal{O}_{\text {s }}$ and two $\mathcal{O}(-1)$ s.

By [11, Lemma 5.3], the locus $\bar{\Sigma}^{1}$ of at least one common zeros is a divisor in $P_{0}$ and the locus of indeterminacy of the birational map [11, (5.30)]

$$
\mathbb{P}^{1} \times \mathrm{P}_{0} \rightarrow \mathbb{P}^{1}
$$

is the normalization $\tilde{\Sigma}$ of $\bar{\Sigma}^{1}$, which is isomorphic to the stable part of $\mathbb{P}^{1} \times$ $\mathbb{P}\left(\operatorname{Sym}^{2}\left(\mathbb{C}^{2}\right) \otimes \mathbb{C}^{2}\right)$ with respect to the linearization $\mathcal{O}(1,1)$. Hence $\mathcal{C}$ is the blow-up of $\mathbb{P}^{1} \times \mathrm{P}_{0}$ along $\tilde{\Sigma}$ and the family of stable maps (4.8) is obtained by the surjective homomorphism

$$
\mathcal{O}_{\mathcal{C}}^{\oplus 2} \longrightarrow \mu^{*} \mathcal{O}_{\mathbb{P}^{1} \times \mathrm{P}_{0}}(3,1) \otimes \mathcal{O}\left(-\mu^{-1}(\tilde{\Sigma})\right)=: \mathrm{H}
$$

induced from the evaluation $\mathcal{O}_{\mathbb{P}^{1} \times \mathrm{P}_{\mathcal{O}}}^{\oplus 2} \rightarrow \mathcal{O}_{\mathbb{P}^{1} \times \mathrm{P}_{0}}(3,1)$. Here $\mu: \mathcal{C} \rightarrow \mathbb{P}^{1} \times \mathrm{P}_{0}$ is the blow-up map. See [11] for more details.

By construction, $\mathrm{ev}^{*} \mathcal{O}_{\mathbb{P}^{1}}(1)=\mathrm{H}$ and from the exact sequence

$$
\left.0 \longrightarrow \mathrm{H} \longrightarrow \mu^{*} \mathcal{O}(3,1) \longrightarrow \mu^{*} \mathcal{O}(3,1)\right|_{\mu^{-1}(\tilde{\Sigma})} \longrightarrow 0
$$

we obtain an exact sequence on $\mathbb{P}^{1} \times \mathrm{P}_{0}$

$$
\left.0 \longrightarrow \mu_{*} \mathrm{H} \longrightarrow \mathcal{O}(3,1) \longrightarrow \mathcal{O}(3,1)\right|_{\tilde{\Sigma}} \longrightarrow 0 \text {. }
$$

Since the pullback of $\mathcal{O}_{\mathrm{P}_{\mathrm{o}}}(1)$ to $\tilde{\Sigma}=\left[\mathbb{P}^{1} \times \mathbb{P}\left(\operatorname{Sym}^{2}\left(\mathbb{C}^{2}\right) \otimes \mathbb{C}^{2}\right)\right]^{\mathrm{s}}$ is $\mathcal{O}(1,1)$, we have

$$
\left.\mathcal{O}(3,1)\right|_{\tilde{\Sigma}} \cong \mathcal{O}_{\mathbb{P}^{1} \times \mathbb{P}^{5}}(4,1) \text {. }
$$

Let $v: \tilde{\Sigma} \rightarrow \bar{\Sigma}^{1}$ be normalization map. Then by taking the direct images with respect to the projection to $\mathrm{P}_{0}$, we obtain an exact sequence

$$
0 \longrightarrow \pi_{*} \mathrm{ev}^{*} \mathcal{O}_{\mathbb{P}^{1}}(1) \longrightarrow \mathcal{O}_{\mathrm{P}_{\mathrm{O}}}(1)^{\oplus 4} \longrightarrow \gamma_{*}\left(\left.\mathcal{O}(3,1)\right|_{\tilde{\Sigma}}\right) \longrightarrow 0
$$

because $\mathrm{R}^{1} \pi_{*} \mathrm{ev}^{*} \mathcal{O}_{\mathbb{P}^{1}}(1)=0$. After tensoring with $\mathcal{O}_{\mathbb{P}^{7}}(\mathrm{k})$, we obtain

$0 \longrightarrow \pi_{*} \mathrm{ev}^{*} \mathcal{O}_{\mathbb{P}^{1}}(1) \otimes \mathcal{O}_{\mathbb{P}^{7}}(\mathrm{k}) \longrightarrow \mathcal{O}_{\mathrm{P}_{\mathrm{O}}}(\mathrm{k}+1)^{\oplus 4} \longrightarrow v_{*}\left(\mathcal{O}_{\mathbb{P}^{1} \times \mathbb{P}^{5}}(\mathrm{k}+4, \mathrm{k}+1)\right) \longrightarrow 0$.

Since the codimension of $\mathbb{P}^{7}-P_{0}$ is 3 , we thus obtain the long exact sequence

$$
\begin{gathered}
0 \longrightarrow \mathrm{H}^{0}\left(\mathbb{P}^{7}, \mathrm{E}(\mathrm{k})\right) \longrightarrow \mathrm{H}^{0}\left(\mathbb{P}^{7}, \mathcal{O}_{\mathbb{P}^{7}}(\mathrm{k}+1)\right)^{\oplus 4} \stackrel{\gamma}{\longrightarrow} \mathrm{H}^{0}\left(\mathbb{P}^{1} \times \mathbb{P}^{5}, \mathcal{O}(\mathrm{k}+4, \mathrm{k}+1)\right) \\
\longrightarrow \mathrm{H}^{1}\left(\mathbb{P}^{7}, \mathrm{E}(\mathrm{k})\right) \longrightarrow \mathrm{H}^{1}\left(\mathbb{P}^{7}, \mathcal{O}_{\mathbb{P}^{7}}(\mathrm{k}+1)\right)^{\oplus 4} \longrightarrow \cdots
\end{gathered}
$$

Clearly, $H^{i}\left(\mathbb{P}^{7}, \mathcal{O}_{\mathbb{P}^{7}}(k+1)\right)^{\oplus 4}=0$ for $i=1,2, \cdots, 6$ and $H^{i}\left(\mathbb{P}^{1} \times \mathbb{P}^{5}, \mathcal{O}(k+4, k+\right.$ $1))=0$ for $i=1,2, \cdots, 5$. Also, by an elementary calculation, we have

$\operatorname{dim} H^{0}\left(\mathbb{P}^{7}, \mathcal{O}_{\mathbb{P}^{7}}(k+1)\right)^{\oplus 4}-\operatorname{dim} H^{0}\left(\mathbb{P}^{1} \times \mathbb{P}^{5}, \mathcal{O}(k+4, k+1)\right)=\operatorname{dim} H^{0}\left(\mathbb{P}^{7}, \mathcal{O}(k) \oplus \mathcal{O}(k-1)\right)^{\oplus 2}$.

Therefore, the lemma follows if $\gamma$ is surjective. Indeed, the space $H^{0}\left(\mathbb{P}^{7}, \mathcal{O}_{\mathbb{P}^{7}}(k+1)\right)$ consists of homogeneous polynomials of degree $k+1$ in eight variables $x_{1}, \cdots, x_{8}$ and $\mathrm{H}^{0}\left(\mathbb{P}^{1} \times \mathbb{P}^{5}, \mathcal{O}(k+4, k+1)\right)$ consists of bihomogeneous polynomials in $z_{0}, z_{1}$ and $y_{1}, \cdots, y_{6}$ of bidegree $(k+4, k+1)$. From the definition of the map $v$, we see that

$$
\begin{aligned}
& x_{1}=z_{0} y_{1}, x_{2}=z_{0} y_{2}+z_{1} y_{1}, x_{3}=z_{0} y_{3}+z_{1} y_{2}, x_{4}=z_{1} y_{3} \\
& x_{5}=z_{0} y_{4}, x_{6}=z_{0} y_{5}+z_{1} y_{4}, x_{7}=z_{0} y_{6}+z_{1} y_{5}, x_{8}=z_{1} y_{6} .
\end{aligned}
$$

By induction on $k$, it is easy to check that $\gamma$ is indeed a surjection. 
Consequently, the normal bundle in $Q_{0}$ of $\mathbb{P}\left(\operatorname{Sym}^{3}\left(\mathbb{C}^{2}\right) \otimes \mathbb{C}^{2}\right)^{s}$, which is a fiber of $\Theta^{1} \rightarrow \mathrm{Gr}(2, \mathrm{r}+1)$, is

$$
\mathcal{O}_{\mathbb{P}^{7}}^{\oplus 2 r-2} \oplus \mathcal{O}_{\mathbb{P}^{7}}(-1)^{\oplus 2 r-2} .
$$

Obviously the factor $\mathcal{O}^{\oplus 2 r-2}$ is the pullback of the tangent space of $\operatorname{Gr}(2, r+1)$ and hence the normal bundle to $\Theta^{1}$ in Qo restricted to $\mathbb{P}\left(\operatorname{Sym}^{3}\left(\mathbb{C}^{2}\right) \otimes \mathbb{C}^{2}\right)^{s}$ is

$$
\mathcal{O}_{\mathbb{P}^{7}}(-1)^{\oplus 2 r-2} \text {. }
$$

We summarize the above discussions as follows.

Corollary 4.3. The indeterminacy locus of $\Phi$ is $\Gamma^{1} \cup \Gamma^{2}$ with $\Gamma^{1}=\Theta^{1} / \operatorname{SL}(2)$, $\Gamma^{2}=\Theta^{2} / \operatorname{SL}(2)$ where $\Theta^{1}$ is a $\mathbb{P}\left(\operatorname{Sym}^{3}\left(\mathbb{C}^{2}\right) \otimes \mathbb{C}^{2}\right)^{s}$-bundle over $\operatorname{Gr}(2, \mathrm{r}+1)$ and $\Theta^{2}$ is a $\mathbb{P}^{r-1}$-bundle over $a\left[\mathbb{P}^{1} \times \mathbb{P}\left(\operatorname{Sym}^{2}\left(\mathbb{C}^{2}\right) \otimes \mathbb{C}^{2}\right)\right]^{\mathrm{s}}$-bundle over $\operatorname{Gr}(2, \mathrm{r}+1)$. The normal bundle to $\Theta^{1}$ in $\mathrm{Q}_{0}$ restricted to $\mathbb{P}\left(\mathrm{Sym}^{3}\left(\mathbb{C}^{2}\right) \otimes \mathbb{C}^{2}\right)^{\mathrm{s}}$ is $\mathcal{O}_{\mathbb{P}^{7}}(-1)^{\oplus 2 \mathrm{r}-2}$.

4.3. Blow-ups. By adjunction, we have a natural homomorphism $\varphi_{f}: \mathcal{O}_{\mathbb{P r}} \rightarrow$ $f_{*} \mathcal{O}_{C}$ for any stable map $f: C \rightarrow \mathbb{P}^{r}$. The image of $\varphi_{f}$ is the structure sheaf $\mathcal{O}_{C^{\prime}}$ of a curve $C^{\prime}$ in $\mathbb{P}^{r}$. If $f \in \Gamma^{1} \cup \Gamma^{2}, \mathcal{O}_{C}$, is a destabilizing subsheaf of $f_{*} \mathcal{O}_{C}$ because

$$
\frac{\chi\left(\mathcal{O}_{\mathrm{C}^{\prime}}(\mathrm{m})\right)}{\mathrm{r}\left(\mathcal{O}_{\mathrm{C}^{\prime}}\right)}=\mathrm{m}+1 \text { or } \frac{2 \mathrm{~m}+1}{2}>\frac{3 \mathrm{~m}+1}{3}=\frac{\chi\left(\mathrm{f}_{*} \mathcal{O}_{\mathrm{C}}(\mathrm{m})\right)}{\mathrm{r}\left(\mathrm{f}_{*} \mathcal{O}_{\mathrm{C}}\right)}
$$

Definition 4.4. A subsheaf $\mathrm{F}$ of a coherent sheaf $\mathrm{E}$ on $\mathbb{P}^{\mathrm{r}}$ is the destabilizing subsheaf if it is the first nonzero term $\mathrm{E}_{1}$ in the Harder-Narasimhan filtration $0 \subset$ $\mathrm{E}_{1} \subset \cdots \subset \mathrm{E}_{\mathrm{k}}=\mathrm{E}$ and $\mathrm{F} \neq \mathrm{E}$. The quotient $\mathrm{E} / \mathrm{F}$ by the destabilizing subsheaf $\mathrm{F}$ is called the destabilizing quotient.

See [9] for fundamental results on Harder-Narasimhan filtration.

Lemma 4.5. $\mathcal{O}_{C^{\prime}}$ is the destabilizing subsheaf of $\mathrm{f}_{*} \mathcal{O}_{\mathrm{C}}$ when $\mathrm{f}_{*} \mathcal{O}_{\mathrm{C}}$ is not stable, i.e. $f \in \Gamma^{1} \cup \Gamma^{2}$.

Proof. For $f \in \Gamma^{1}, f_{*} \mathcal{O}_{C}$ is supported on a line $L$ in $\mathbb{P}^{r}$ and hence $f_{*} \mathcal{O}_{C} \cong \mathcal{O}_{\mathrm{L}}(\mathrm{a}) \oplus$ $\mathcal{O}_{\mathrm{L}}(\mathrm{b}) \oplus \mathcal{O}_{\mathrm{L}}(\mathrm{c})$ for $\mathrm{a} \geq \mathrm{b} \geq \mathrm{c}$. Since the Hilbert polynomial of $\mathrm{f}_{*} \mathcal{O}_{\mathrm{C}}$ is $3 \mathrm{~m}+1$, $\mathrm{a}+\mathrm{b}+\mathrm{c}=-2$. Also, $\mathrm{f}_{*} \mathcal{O}_{\mathrm{c}}$ admits a unique section $\varphi_{\mathrm{f}}$ and hence $\mathrm{a}=0, \mathrm{~b}=$ $-1, c=-1$. Therefore, the image $\mathcal{O}_{\mathrm{C}^{\prime}}$ of $\varphi_{\mathrm{f}}$ is the destabilizing subsheaf $\mathcal{O}_{\mathrm{L}}$.

For $f \in \Gamma^{2}-\Gamma^{1}, C^{\prime}$ is the union of two lines $L_{1}$ and $L_{2}$ such that $\left.f_{*} \mathcal{O}_{C}\right|_{L_{1}} \cong \mathcal{O}_{L_{1}}$ and $\left.\mathrm{f}_{*} \mathcal{O}_{\mathrm{C}}\right|_{\mathrm{L}_{2}} \cong \mathcal{O}_{\mathrm{L}_{2}} \oplus \mathcal{O}_{\mathrm{L}_{2}}(-1)$. As $\mathcal{O}_{\mathrm{C}^{\prime}}$ is the gluing of $\mathcal{O}_{\mathrm{L}_{1}}$ and $\mathcal{O}_{\mathrm{L}_{2}}$ at a point, this is certainly the destabilizing subsheaf of $f_{*} \mathcal{O}_{\mathrm{C}}$.

From the above proof, we see that when $f \in \Gamma^{1}$,

$$
f_{*} \mathcal{O}_{\mathrm{C}} \cong \mathcal{O}_{\mathrm{L}} \oplus \mathcal{O}_{\mathrm{L}}(-1) \oplus \mathcal{O}_{\mathrm{L}}(-1)
$$

for a line $L=f(C)$ in $\mathbb{P}^{r}$. Note that the direct image sheaf depends only on the image line $\mathrm{L}$.

Let $\mathrm{q}_{1}: \mathrm{Q}_{1} \rightarrow \mathrm{Q}_{0}$ be the blow-up along the smooth subvariety $\Theta^{1}$ and let $p_{1}: \mathbf{M}_{1} \rightarrow \mathbf{M}$ be the quotient, i.e. the blow-up along the subvariety $\Gamma^{1}$ ([13, Lemma 3.11]). Let $\Theta_{1}^{1}$ be the exceptional divisor and $\Theta_{1}^{2}$ be the proper transform of $\Theta^{2}$. Let $\Gamma_{1}^{i}$ be the quotient of $\Theta_{1}^{i}$ by $\operatorname{SL}(2)$. We pull back $\varphi_{\mathrm{f}}$ to $\mathbb{P}^{r} \times \mathrm{Q}_{1}$ and restrict it to the divisor $\mathbb{P}^{r} \times \Theta_{1}^{1}$. Let $A_{1}$ be its cokernel on $\mathbb{P}^{r} \times \Theta_{1}^{1}$. Let $\mathcal{E}_{1}$ be the kernel of the composition

$$
\left.\left(1 \times \mathrm{q}_{1}\right)^{*} \mathcal{E}_{0} \longrightarrow\left(1 \times \mathrm{q}_{1}\right)^{*} \mathcal{E}_{0}\right|_{\mathbb{P r} \times \Theta_{1}^{1}} \longrightarrow A_{1}
$$


Lemma 4.6. (1) $\mathcal{E}_{1}$ is a flat family of coherent sheaves on $\mathbb{P}^{\mathrm{r}}$ parameterized by $\mathrm{Q}_{1}$.

(2) The locus where $\mathcal{E}_{1}$ is not stable has two irreducible components $\Theta_{1}^{2} \cup \Theta_{1}^{3}$ where $\Theta_{1}^{3}$ is a smooth subvariety of $\Theta_{1}^{1}$, which is a $\mathbb{P}^{1} \times \mathbb{P}^{r-2}$-bundle over $\Theta^{1}$.

Proof. Let $f: C \rightarrow L \subset \mathbb{P}^{r}$ be a point in $\Theta^{1}$. If we denote the graph of $f$ in $C \times \mathbb{P}^{r}$ by $G$, it is well known that the deformation space of the map $f$ with $C$ fixed is the same as the tangent space at $\mathrm{G}$

$$
\operatorname{Hom}_{\mathrm{C} \times \mathbb{P r}^{\mathrm{P}}}\left(\mathrm{I}_{\mathrm{G}}, \mathcal{O}_{\mathrm{G}}\right)
$$

of the Hilbert scheme of closed subschemes in $C \times \mathbb{P}^{r}$ where $I_{G}$ is the ideal sheaf of G. From the short exact sequence

$$
0 \longrightarrow \mathrm{I}_{\mathrm{G}} \longrightarrow \mathcal{O}_{\mathrm{C} \times \mathbb{P}^{r}} \longrightarrow \mathcal{O}_{\mathrm{G}} \longrightarrow 0
$$

we obtain an isomorphism

$$
\operatorname{Hom}\left(\mathrm{I}_{\mathrm{G}}, \mathcal{O}_{\mathrm{G}}\right) \cong \operatorname{Ext}_{\mathrm{C} \times \mathbb{P}^{r}}^{1}\left(\mathcal{O}_{\mathrm{G}}, \mathcal{O}_{\mathrm{G}}\right)
$$

because $H^{1}\left(\mathcal{O}_{G}\right)=H^{1}\left(\mathcal{O}_{C}\right)=0$. Since $\left.I_{G}\right|_{G} \cong f^{*} \Omega_{\mathbb{P r}}$ and $\mathcal{O}_{G} \cong \mathcal{O}_{C}$, we have isomorphisms

$$
\operatorname{Hom}\left(\mathrm{I}_{\mathrm{G}}, \mathcal{O}_{\mathrm{G}}\right) \cong \operatorname{Hom}_{\mathrm{C}}\left(f^{*} \Omega_{\mathbb{P}^{r}}, \mathcal{O}_{\mathrm{C}}\right) \cong \operatorname{Hom}_{\mathbb{P}^{r}}\left(\Omega_{\mathbb{P}^{r}}, f_{*} \mathcal{O}_{\mathrm{C}}\right) .
$$

The natural morphism $\mathrm{I}_{\mathrm{L}} \stackrel{\mathrm{d}}{\longrightarrow} \Omega_{\mathbb{P r}}$ and the projection $\mathrm{f}_{*} \mathcal{O}_{\mathrm{C}} \rightarrow \mathcal{O}_{\mathrm{L}}(-1)^{\oplus 2}$ by $(4.9)$ induce the homomorphisms

$$
\operatorname{Hom}\left(\Omega_{\mathbb{P}^{r}}, \mathrm{f}_{*} \mathcal{O}_{\mathrm{C}}\right) \longrightarrow \operatorname{Hom}\left(\mathrm{I}_{\mathrm{L}}, \mathrm{f}_{*} \mathcal{O}_{\mathrm{C}}\right) \longrightarrow \operatorname{Hom}\left(\mathrm{I}_{\mathrm{L}}, \mathcal{O}_{\mathrm{L}}(-1)\right)^{2}
$$

where $I_{L}$ is the ideal sheaf of $L$ on $\mathbb{P}^{r}$. On the other hand, as $R^{1} p_{*} \mathcal{O}_{G}=0$ where $p: C \times \mathbb{P}^{r} \rightarrow \mathbb{P}^{r}$ is the projection, an extension of $\mathcal{O}_{\mathrm{G}}$ by itself gives us an extension of $p_{*} \mathcal{O}_{\mathrm{G}} \cong f_{*} \mathcal{O}_{\mathrm{C}}$ by itself. Thus we have homomorphisms

$\operatorname{Ext}^{1}\left(\mathcal{O}_{\mathrm{G}}, \mathcal{O}_{\mathrm{G}}\right) \longrightarrow \operatorname{Ext}^{1}\left(\mathrm{f}_{*} \mathcal{O}_{\mathrm{C}}, \mathrm{f}_{*} \mathcal{O}_{\mathrm{C}}\right) \longrightarrow \operatorname{Ext}^{1}\left(\mathcal{O}_{\mathrm{L}}, \mathrm{f}_{*} \mathcal{O}_{\mathrm{C}}\right) \longrightarrow \operatorname{Ext}^{1}\left(\mathcal{O}_{\mathrm{L}}, \mathcal{O}_{\mathrm{L}}(-1)\right)^{2}$

where the last two come from (4.9). Then it is an easy exercise to check the commutativity of the following diagram

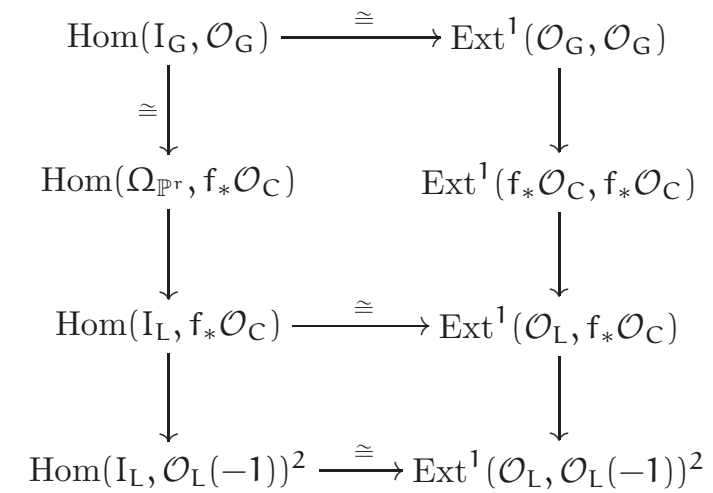

where the last two horizontal maps are from the exact sequence $0 \rightarrow \mathrm{I}_{\mathrm{L}} \rightarrow \mathcal{O}_{\mathbb{P r}} \rightarrow$ $\mathcal{O}_{\mathrm{L}} \rightarrow 0$.

From the exact sequence

$$
0 \longrightarrow \mathrm{I}_{\mathrm{L}} /\left.\mathrm{I}_{\mathrm{L}}^{2} \longrightarrow \Omega_{\mathbb{P}^{r}}\right|_{\mathrm{L}} \longrightarrow \Omega_{\mathrm{L}} \longrightarrow 0
$$


we see that the normal space to the deformation space of $f$ as a map to $L$ in the deformation space of $f$ as a map to $\mathbb{P}^{r}$ is exactly $\operatorname{Hom}\left(I_{L}, f_{*} \mathcal{O}_{C}\right)$. Furthermore, the tangent space to the Grassmannian is exactly $\operatorname{Hom}\left(\mathrm{I}_{\mathrm{L}}, \mathcal{O}_{\mathrm{L}}\right)$ and hence the normal space of $\Theta^{1}$ in $Q_{0}$ at $f$ is

$$
\mathrm{N}_{\Theta^{1} / \mathrm{Q}_{0}, \mathrm{f}}=\operatorname{Hom}\left(\mathrm{I}_{\mathrm{L}}, \mathcal{O}_{\mathrm{L}}(-1)\right)^{2}
$$

which is the bottom left term in (4.10).

The Kodaira-Spencer map for the family $\mathcal{E}_{0}$ of sheaves

$$
\mathrm{T}_{\mathrm{f}} \mathrm{Q}_{\mathrm{O}} \rightarrow \operatorname{Ext}_{\mathbb{P r}}^{1}\left(\mathrm{f}_{*} \mathcal{O}_{\mathrm{C}}, \mathrm{f}_{*} \mathcal{O}_{\mathrm{C}}\right)
$$

sends any extension $\tilde{f}: \tilde{C}=C \times \operatorname{Spec} \mathbb{C}[\epsilon] /\left(\epsilon^{2}\right) \longrightarrow \mathbb{P}^{r}$ of $f$ to the extension class of $\mathrm{O} \longrightarrow \epsilon \cdot \mathrm{f}_{*} \mathcal{O}_{\mathrm{C}} \longrightarrow \tilde{f}_{*} \mathcal{O}_{\tilde{\mathrm{C}}} \longrightarrow \mathrm{f}_{*} \mathcal{O}_{\mathrm{C}} \longrightarrow 0$ obtained from the obvious extension $0 \longrightarrow \epsilon \mathcal{O}_{\mathrm{C}} \longrightarrow \mathcal{O}_{\tilde{\mathrm{C}}} \longrightarrow \mathcal{O}_{\mathrm{C}} \longrightarrow 0$ since $\mathrm{R}^{1} \mathrm{f}_{*} \mathcal{O}_{\mathrm{C}}=0$. By $(4.9), \mathrm{f}_{*} \mathcal{O}_{\mathrm{C}}$ remains fixed along the fibers of $\Theta^{1} \rightarrow \operatorname{Gr}(2, r+1)$ and the variation of $L$ is sent to the factor $\operatorname{Ext}^{1}\left(\mathcal{O}_{\mathrm{L}}, \mathcal{O}_{\mathrm{L}}\right)$. Hence we have an induced homomorphism

$$
\mathrm{N}_{\Theta^{1} / \mathrm{Q}_{0}, \mathrm{f}}=\operatorname{Hom}\left(\mathrm{I}_{\mathrm{L}}, \mathcal{O}_{\mathrm{L}}(-1)\right)^{2} \longrightarrow \operatorname{Ext}^{1}\left(\mathcal{O}_{\mathrm{L}}, \mathcal{O}_{\mathrm{L}}(-1)\right)^{2}
$$

by the projection $\operatorname{Ext}_{\mathbb{P r}}^{1}\left(f_{*} \mathcal{O}_{\mathrm{C}}, f_{*} \mathcal{O}_{\mathrm{C}}\right) \rightarrow \operatorname{Ext}^{1}\left(\mathcal{O}_{\mathrm{L}}, \mathcal{O}_{\mathrm{L}}(-1)\right)^{2}$. From the above discussions, we see that the horizontal maps in (4.10) give us the Kodaira-Spencer map and hence (4.12) is an isomorphism.

Let us consider the effect of elementary modification $\mathcal{E}_{1}$. Choosing a point in $\mathrm{Q}_{1}$ lying over $f$ is the same as choosing a normal vector to $\Theta^{1}$ at $f$. Over $\operatorname{Spec} \mathbb{C}[\epsilon] /\left(\epsilon^{2}\right)$, the process of taking $\mathcal{E}_{1}$ from $\mathcal{E}_{0}$ is explained in the diagram

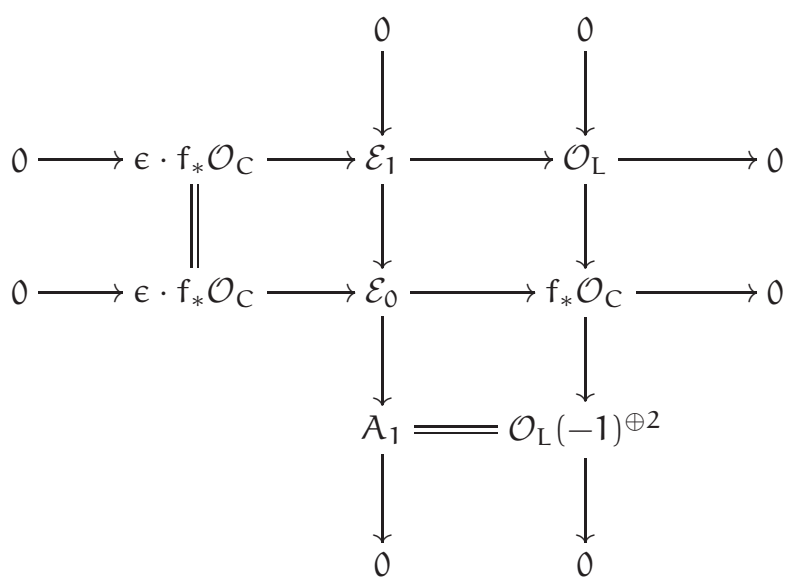

Since $\mathrm{f}_{*} \mathcal{O}_{\mathrm{C}}=\mathcal{O}_{\mathrm{L}} \oplus \mathcal{O}_{\mathrm{L}}(-1)^{\oplus 2}$, the central fiber $\mathcal{E}_{1} / \epsilon \mathcal{E}_{1}$ fits into an exact sequence

$$
0 \longrightarrow \mathcal{O}_{\mathrm{L}}(-1)^{\oplus 2} \longrightarrow \mathcal{E}_{1} / \epsilon \mathcal{E}_{1} \longrightarrow \mathcal{O}_{\mathrm{L}} \longrightarrow 0
$$

whose extension class is the image of (4.12). In particular, $f_{*} \mathcal{O}_{\mathrm{C}} \cong \mathcal{O}_{\mathrm{L}} \oplus \mathcal{O}_{\mathrm{L}}(-1) \oplus$ $\mathcal{O}_{\mathrm{L}}(-1)$ and $\left.\mathcal{E}_{1}\right|_{\mathbb{P}^{\mathrm{r}} \times\{\mathrm{y}\}}$ is an extension of $\mathcal{O}_{\mathrm{L}}$ by $\mathcal{O}_{\mathrm{L}}(-1) \oplus \mathcal{O}_{\mathrm{L}}(-1)$ for any $\mathrm{y} \in \mathrm{Q}_{1}$ lying over $f$. In particular, the Hilbert polynomial is constantly $3 m+1$ and hence $\mathcal{E}_{1}$ is flat over $\mathrm{Q}_{1}$. Each element in $\mathbb{P N}_{\Theta^{1} / Q_{0}, f} \cong \mathbb{P}\left(\operatorname{Ext}_{\mathbb{P}^{r}}^{1}\left(\mathcal{O}_{\mathrm{L}}, \mathcal{O}_{\mathrm{L}}(-1)\right) \otimes \mathbb{C}^{2}\right)$ parameterizes an extension

$$
0 \rightarrow \mathcal{O}_{\mathrm{L}}(-1) \oplus \mathcal{O}_{\mathrm{L}}(-1) \rightarrow \mathrm{E} \rightarrow \mathcal{O}_{\mathrm{L}} \rightarrow 0
$$


which is unstable if and only if it belongs to $\operatorname{PExt}_{\mathbb{P r}}^{1}\left(\mathcal{O}_{\mathrm{L}}, \mathcal{O}_{\mathrm{L}}(-1)\right) \times \mathbb{P}^{1}$, in which case $\mathrm{E}=\mathrm{F} \oplus \mathcal{O}_{\mathrm{L}}(-1)$ for a stable sheaf $\mathrm{F}$ which is a nontrivial extension of $\mathcal{O}_{\mathrm{L}}$ by $\mathcal{O}_{\mathrm{L}}(-1)$.

Example 4.7. Let $f: \mathbb{P}^{1} \rightarrow \mathbb{P}^{r}$ be a stable map given by $\left(z_{0}^{3}: z_{1}^{3}: 0: \cdots: 0\right)$ and consider a deformation of $f$ over $\operatorname{Spec} \mathbb{C}[\epsilon] /\left(\epsilon^{2}\right)$ given by the map

$$
\left(z_{0}^{3}: z_{1}^{3}: \epsilon z_{0}^{2} z_{1}: \epsilon z_{0} z_{1}^{2}: 0: \cdots: 0\right) \text {. }
$$

In affine charts, $\mathcal{E}_{0}=\mathrm{f}_{*} \mathcal{O}_{\mathrm{C}}$ is $\mathbb{C}[\mathrm{t}, \epsilon]$ which is a module over $\mathbb{C}\left[x_{1}, x_{2}, \cdots, x_{\mathrm{r}}\right]$ by

$$
x_{1}=t^{3}, \quad x_{2}=\epsilon t, \quad x_{3}=\epsilon t^{2}, \quad x_{4}=\cdots=x_{r}=0
$$

Then the central fiber of $\mathcal{E}_{0}$ is

$$
\mathcal{E}_{0} / \epsilon \mathcal{E}_{0}=\mathbb{C}[\mathrm{t}] \cong \mathbb{C}\left[x_{1}\right] \oplus \mathrm{t} \mathbb{C}\left[x_{1}\right] \oplus \mathrm{t}^{2} \mathbb{C}\left[x_{1}\right]
$$

and the destabilizing subsheaf is $\mathbb{C}\left[x_{1}\right]$. Hence we have

$$
\mathcal{E}_{1}=\operatorname{ker}\left(\mathbb{C}[\mathrm{t}, \epsilon] \longrightarrow \mathbb{C}[\mathrm{t}] \longrightarrow \mathrm{t} \mathbb{C}\left[x_{1}\right] \oplus \mathrm{t}^{2} \mathbb{C}\left[x_{1}\right]\right)=\left\{\mathrm{g}\left(\mathrm{x}_{1}\right)+\epsilon \mathrm{h}(\mathrm{t}, \epsilon)\right\}
$$

where $\mathrm{g}, \mathrm{h}$ are polynomials. Hence the central fiber of $\mathcal{E}_{1}$ is

$$
\begin{gathered}
\mathcal{E}_{1} / \epsilon \mathcal{E}_{1}=\left\{g\left(x_{1}\right)+\epsilon \operatorname{tg}_{1}\left(x_{1}\right)+\epsilon t^{2} g_{2}\left(x_{1}\right)\right\} \cong \mathbb{C}\left[x_{1}\right] \oplus x_{2} \mathbb{C}\left[x_{1}\right] \oplus x_{3} \mathbb{C}\left[x_{1}\right] \\
\cong \mathbb{C}\left[x_{1}, x_{2}, \cdots, x_{r}\right] /\left(x_{2}^{2}, x_{2} x_{3}, x_{3}^{2}, x_{4}, \cdots, x_{r}\right)
\end{gathered}
$$

i.e. the thickening of the line $x_{2}=x_{3}=\cdots=0$ in $\mathbb{P}^{3}$ given by $x_{4}=\cdots=0$.

Remark 4.8. By Corollary $4.3, \Theta_{1}^{1}$ is a $\mathbb{P}^{2 r-3} \times\left(\mathbb{P}^{7}\right)^{s}$ bundle over $\mathrm{Gr}(2, \mathrm{r}+1)$. The proof of Lemma 4.6 tells us that the family of stable sheaves $\mathcal{E}_{1}$ over $\left(\mathbb{P}^{2 r-3}-\right.$ $\left.\mathbb{P}^{1} \times \mathbb{P}^{r-2}\right) \times\left(\mathbb{P}^{7}\right)^{s}$ remains constant for the $\left(\mathbb{P}^{7}\right)^{s}$ direction and depends only on the extension classes in $\mathbb{P}^{2 r-3}=\mathbb{P E x t}^{1}\left(\mathcal{O}_{\mathrm{L}}, \mathcal{O}_{\mathrm{L}}(-1)\right)^{2}$.

By Lemma 4.6, we have an invariant morphism

$$
\Psi_{1}: Q_{1}-\Theta_{1}^{2} \cup \Theta_{1}^{3} \longrightarrow \mathbf{S}
$$

which induces a morphism

$$
\Phi_{1}: \mathbf{M}_{1}-\Gamma_{1}^{2} \cup \Gamma_{1}^{3} \longrightarrow \mathbf{S}
$$

where $\Gamma_{1}^{i}$ is the quotient of $\Theta_{1}^{i}$.

Let $\mathrm{q}_{2}: \mathrm{Q}_{2} \rightarrow \mathrm{Q}_{1}$ be the blow-up along $\Theta_{1}^{2}$ and $\mathrm{p}_{2}: \mathbf{M}_{2} \rightarrow \mathbf{M}_{1}$ be its quotient by $\operatorname{SL}(2)$. Let $\Theta_{2}^{2}$ be the exceptional divisor and $\Theta_{2}^{1}, \Theta_{2}^{3}$ be the proper transforms of $\Theta_{1}^{1}, \Theta_{1}^{3}$. Let $\Gamma_{2}^{i}$ be the quotient of $\Theta_{2}^{i}$ by $\operatorname{SL}(2)$. Let $\mathcal{E}_{2}^{\prime}$ be the pull-back of $\mathcal{E}_{1}$ to $\mathbb{P}^{\mathrm{r}} \times \mathrm{Q}_{2}$.

Lemma 4.9. There is a unique flat family of quotient sheaves on the divisor $\Theta_{2}^{2}$

$$
\left.\mathcal{E}_{2}^{\prime}\right|_{\mathbb{P r}^{r} \times \Theta_{2}^{2}} \rightarrow A_{2}
$$

such that the Hilbert polynomial of $\mathrm{A}_{2}$ at every point of $\Theta_{2}^{2}$ is $\mathrm{m}$.

Proof. To prove this claim, it suffices to show that for any $y \in \Theta_{2}^{2},\left.\mathcal{E}_{2}^{\prime}\right|_{\mathbb{P r}^{\mathrm{r}} \times\{y\}}$ has a destabilizing subsheaf of Hilbert polynomial $2 m+1$. From the uniqueness of the Harder-Narasimhan filtration and the existence of relative Quot scheme [9, Chapter $2]$, we deduce that there is such a flat quotient $A_{2}$.

Let $f \in \Theta^{2}-\Theta^{1}$, i.e. $f: C \rightarrow \mathbb{P}^{r}$ is a stable map whose image is the union $C^{\prime}$ of two distinct lines $L_{1}$ and $L_{2}$. Without loss of generality, we may assume $L_{2}$ is 
the degree 2 component. By adjunction, we have a subsheaf $\mathcal{O}_{C^{\prime}}$ of $f_{*} \mathcal{O}_{C}$ and a nonsplit extension

$$
0 \rightarrow \mathcal{O}_{\mathrm{C}^{\prime}} \rightarrow \mathrm{f}_{*} \mathcal{O}_{\mathrm{C}} \rightarrow \mathcal{O}_{\mathrm{L}_{2}}(-1) \rightarrow 0 .
$$

Since $\mathcal{O}_{\mathrm{C}^{\prime}}$ and $\mathcal{O}_{\mathrm{L}_{2}}(-1)$ are stable, the destabilizing subsheaf of $f_{*} \mathcal{O}_{\mathrm{C}}$ is $\mathcal{O}_{\mathrm{C}^{\prime}}$, whose Hilbert polynomial is $2 m+1$.

From Lemma 4.6, we see that $\Theta_{1}^{2} \cap \Theta_{1}^{1} \subset \Theta_{1}^{3}$. Suppose $y \in \Theta_{1}^{2} \cap \Theta_{1}^{3}$. Then $q_{1}(y)$ is represented by a stable map $f: C \rightarrow L \subset \mathbb{P}^{r}$ where $C=C_{1} \cup C_{2}$ is reducible and $L$ is a line. From the proof of Lemma 4.6, one can check that $q_{1}^{-1}(f) \cap \Theta_{1}^{2}=$ $\mathbb{P} \operatorname{Ext}^{1}\left(\mathcal{O}_{\mathrm{L}}, \mathcal{O}_{\mathrm{L}}(-1)\right)$ and $\left.\mathcal{E}_{1}\right|_{\mathbb{P r}^{r} \times\{y\}} \cong \mathrm{F} \oplus \mathcal{O}_{\mathrm{L}}(-1)$ where $\mathrm{F}$ is a nonsplit extension of $\mathcal{O}_{\mathrm{L}}$ by $\mathcal{O}_{\mathrm{L}}(-1)$. Hence, $\mathrm{F}$ is the destabilizing subsheaf of $\left.\mathcal{E}_{1}\right|_{\mathbb{P r}^{\mathrm{r}} \times\{y\}}$ whose Hilbert polynomial is $2 \mathrm{~m}+1$.

Let $\mathcal{E}_{2}$ be the kernel of the composition

$$
\left.\mathcal{E}_{2}^{\prime} \rightarrow \mathcal{E}_{2}^{\prime}\right|_{\mathbb{P}^{r} \times \Theta_{2}^{2}} \rightarrow \mathrm{A}_{2} .
$$

Lemma 4.10. (1) $\mathcal{E}_{2}$ is a flat family of coherent sheaves on $\mathbb{P}^{r}$ parameterized by $\mathrm{Q}_{2}$.

(2) The locus of unstable sheaves is precisely $\Theta_{2}^{3}$ and we have an invariant morphism

$$
\Psi_{2}: \mathrm{Q}_{2}-\Theta_{2}^{3} \longrightarrow \mathbf{S}
$$

which induces a morphism

$$
\Phi_{2}: \mathbf{M}_{2}-\Gamma_{2}^{3} \longrightarrow \mathbf{S}
$$

Proof. As we saw in the proof of Lemma 4.6, the effect of elementary modification is interchanging the destabilizing subsheaf with the quotient. For $y \in \Theta_{2}^{2}-\mathrm{q}_{2}^{-1}\left(\Theta_{1}^{3}\right)$, $\mathcal{E}_{2}{\mid \mathbb{P r}^{\mathrm{r}} \times\{y\}}_{\text {is }}$ an extension

$$
\left.0 \rightarrow \mathcal{O}_{\mathrm{L}_{2}}(-1) \rightarrow \mathcal{E}_{2}\right|_{\mathbb{P r}^{r} \times\{y\}} \rightarrow \mathcal{O}_{\mathrm{C}^{\prime}} \rightarrow 0
$$

and thus the Hilbert polynomial remains unchanged. We claim $E=\left.\mathcal{E}_{2}\right|_{\mathbb{P r}^{r} \times\{y\}}$ is stable. Indeed, (4.13) gives an exact sequence

$$
0 \rightarrow \mathrm{N}_{\mathrm{C}^{\prime}} \rightarrow \mathrm{N}_{\mathrm{C}^{\prime}} \otimes \mathrm{f}_{*} \mathcal{O}_{\mathrm{C}} \cong \mathrm{f}_{*} \mathrm{f}^{*} \mathrm{~N}_{\mathrm{C}^{\prime}} \rightarrow \mathcal{O}_{\mathrm{L}_{2}}(-1) \otimes \mathrm{N}_{\mathrm{C}^{\prime}} \rightarrow 0
$$

by tensoring the normal bundle $\mathrm{N}_{\mathrm{C}^{\prime}}$ of the complete intersection $\mathrm{C}^{\prime}$. Upon taking cohomology, we get a diagram

$$
0 \longrightarrow \mathrm{H}^{0}\left(\mathrm{C}^{\prime}, \mathrm{N}_{\mathrm{C}^{\prime}}\right) \longrightarrow \mathrm{H}^{0}\left(\mathrm{C}^{\prime}, \mathrm{f}_{*} \mathrm{f}^{*} \mathrm{~N}_{\mathrm{C}^{\prime}}\right) \longrightarrow \mathrm{H}^{0}\left(\mathrm{C}^{\prime}, \mathcal{O}_{\mathrm{L}_{2}}(-1) \otimes \mathrm{N}_{\mathrm{C}^{\prime}}\right) \longrightarrow 0
$$

The deformation of $\mathrm{C}^{\prime}$ while keeping a node is parameterized by the kernel of

$$
\mathrm{H}^{0}\left(\mathrm{C}^{\prime}, \mathrm{N}_{\mathrm{C}^{\prime}}\right) \longrightarrow \operatorname{Ext}^{1}\left(\Omega_{\mathrm{C}^{\prime}}, \mathcal{O}_{\mathrm{C}^{\prime}}\right) \cong \mathbb{C}
$$

which comes from the exact sequence

$$
\left.0 \rightarrow \mathrm{N}_{\mathrm{C}^{\prime}}^{*} \rightarrow \Omega_{\mathbb{P} r}\right|_{\mathrm{C}^{\prime}} \rightarrow \Omega_{\mathrm{C}^{\prime}} \rightarrow 0 .
$$

Here, $\operatorname{Ext}^{1}\left(\Omega_{C^{\prime}}, \mathcal{O}_{C^{\prime}}\right) \cong \mathbb{C}$ is the smoothing direction of the node of $C^{\prime}$.

Next, we calculate the quotient

$$
\mathrm{T}_{\mathrm{f}} \mathcal{M}_{0}\left(\mathbb{P}^{\mathrm{r}}, 3\right) / \mathrm{T}_{\mathrm{f}} \mathcal{M}_{\mathrm{o}}\left(\mathrm{C}^{\prime}, 3\right) \text {. }
$$

It is well known that the tangent space of $\mathcal{M}_{0}\left(\mathbb{P}^{r}, 3\right)\left(\operatorname{resp} . \mathcal{M}_{0}\left(C^{\prime}, 3\right)\right)$ is given by the hypercohomology

$$
\operatorname{Ext}^{1}\left(\left\{f^{*} \Omega_{\mathbb{P}^{r}} \rightarrow \mathcal{O}_{C}\right\}, \mathcal{O}_{C}\right) \quad\left(\text { resp. } \operatorname{Ext}^{1}\left(\left\{f^{*} \Omega_{C^{\prime}} \rightarrow \mathcal{O}_{C}\right\}, \mathcal{O}_{C}\right)\right) .
$$


By applying the octahedron axiom for the derived category of sheaf complexes on $\mathrm{C}$ to the composition $\mathrm{f}^{*} \Omega_{\mathbb{P}^{r}} \rightarrow \mathrm{f}^{*} \Omega_{\mathrm{C}^{\prime}} \rightarrow \mathcal{O}_{\mathrm{C}}$, we obtain a distinguished triangle

$$
f^{*} N_{C^{\prime}}^{*}[1] \longrightarrow\left\{f^{*} \Omega_{\mathbb{P}^{r}} \rightarrow \mathcal{O}_{\mathrm{C}}\right\} \longrightarrow\left\{f^{*} \Omega_{\mathrm{C}^{\prime}} \rightarrow \mathcal{O}_{\mathrm{C}}\right\} \stackrel{[1]}{\longrightarrow} .
$$

This induces an exact sequence

$0 \longrightarrow \mathrm{T}_{\mathrm{f}} \mathcal{M}_{\mathrm{o}}\left(\mathrm{C}^{\prime}, 3\right) \longrightarrow \mathrm{T}_{\mathrm{f}} \mathcal{M}_{\mathrm{o}}\left(\mathbb{P}^{\mathrm{r}}, 3\right) \longrightarrow \mathrm{H}^{\mathrm{0}}\left(\mathrm{C}, \mathrm{f}^{*} \mathrm{~N}_{\mathrm{C}^{\prime}}\right) \longrightarrow \operatorname{Ext}^{2}\left(\left\{\mathrm{f}^{*} \Omega_{\mathrm{C}^{\prime}} \rightarrow \mathcal{O}_{\mathrm{C}}\right\}, \mathcal{O}_{\mathrm{C}}\right) \longrightarrow 0$.

Since the dimension of $\mathcal{M}_{0}\left(C^{\prime}, 3\right)\left(\operatorname{resp} . \mathcal{M}_{0}\left(\mathbb{P}^{r}, 3\right)\right)$ at $f$ is 2 (resp. 4r) and the dimension of $\mathrm{H}^{0}\left(\mathrm{C}, \mathrm{f}^{*} \mathrm{~N}_{\mathrm{C}^{\prime}}\right)$ is $7+4(\mathrm{r}-2)$ by $\mathrm{N}_{\mathrm{C}^{\prime}}=\mathcal{O}(2) \oplus \mathcal{O}(1)^{\mathrm{r}-2}$, we have $\operatorname{Ext}^{2}\left(\left\{f^{*} \Omega_{C^{\prime}} \rightarrow \mathcal{O}_{C}\right\}, \mathcal{O}_{C}\right) \cong \mathbb{C}$. Thus we have a commutative diagram

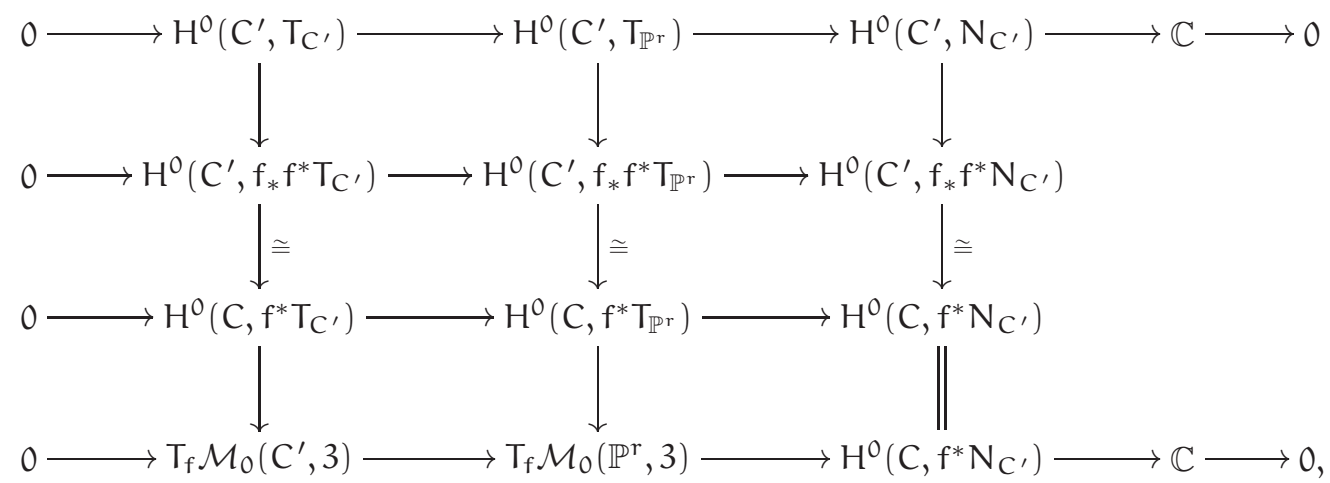

which induces a commutative diagram

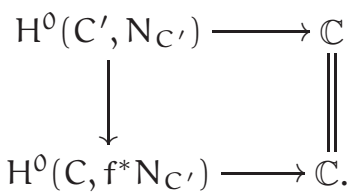

The kernel of the top row is the deformation of $C^{\prime}$ and the kernel of the bottom row is the quotient $T_{f} \mathcal{M}_{0}\left(\mathbb{P}^{r}, 3\right) / T_{f} \mathcal{M}_{0}\left(C^{\prime}, 3\right)$. Therefore, by $(4.15)$ the normal space to $\Theta_{1}^{2}$ at $q_{2}(y)$ in $Q_{1}$ is isomorphic to

$$
H^{0}\left(C^{\prime}, \mathcal{O}_{L_{2}}(-1) \otimes N_{C^{\prime}}\right) \cong \operatorname{Hom}\left(\mathcal{I}_{\mathrm{C}^{\prime}}, \mathcal{O}_{\mathrm{L}_{2}}(-1)\right) \cong \operatorname{Ext}^{1}\left(\mathcal{O}_{\mathrm{C}^{\prime}}, \mathcal{O}_{\mathrm{L}_{2}}(-1)\right)
$$

where $\mathcal{I}_{C^{\prime}}$ is the ideal sheaf of $C^{\prime}$. As in the proof of Lemma 4.6, one can check that this isomorphism is compatible with the Kodaira-Spencer map for $\mathcal{E}_{1}$ and thus (4.14) is nonsplit. Stability follows immediately.

Next suppose $y \in q_{2}^{-1}\left(\Theta_{1}^{3}\right) \cap \Theta_{2}^{2}$. Let $y_{1}=q_{2}(y) \in \Theta_{1}^{3}$ and $y_{0}=q_{1}\left(y_{1}\right)$ be a stable map $\mathrm{f}: \mathrm{C} \rightarrow \mathrm{L} \subset \mathbb{P}^{\mathrm{r}}$ for some line $\mathrm{L}$. Then $\mathrm{q}_{1}^{-1}\left(\mathrm{y}_{0}\right) \cap \Theta_{1}^{2} \cong$ $\mathbb{P} \operatorname{Ext}^{1}\left(\mathcal{O}_{\mathrm{L}}, \mathcal{O}_{\mathrm{L}}(-1)\right)=\mathbb{P}^{\mathrm{r}-2}$. From the proof of Lemma 4.6 , we see that the normal space to $\Theta_{1}^{2}$ at $y_{1}$ is $\operatorname{Ext}^{1}\left(\mathcal{O}_{\mathrm{L}}, \mathcal{O}_{\mathrm{L}}(-1)\right) \oplus \mathbb{C}$ where the summand $\mathbb{C}$ parameterizes the smoothing of $C$, i.e. it comes from the normal direction of $\Theta^{1} \cap \Theta^{2}$ in $\Theta^{1}$. Since $y_{1} \in q_{1}^{-1}\left(y_{0}\right) \cap \Theta_{1}^{2}=\mathbb{P E x t}^{1}\left(\mathcal{O}_{\mathrm{L}}, \mathcal{O}_{\mathrm{L}}(-1)\right), \mathrm{y}_{1}$ can be thought of as a line in $\operatorname{Ext}^{1}\left(\mathcal{O}_{\mathrm{L}}, \mathcal{O}_{\mathrm{L}}(-1)\right)$. Certainly, the isomorphism classes of sheaves in the family $\mathcal{E}_{1}$ don't change in the smoothing direction and along the line of $y_{1}$. Hence the Kodaira-Spencer map on the normal space to $\Theta_{1}^{2}$ factors through $\operatorname{Ext}^{1}\left(\mathcal{O}_{\mathrm{L}}, \mathcal{O}_{\mathrm{L}}(-1)\right) / \mathbb{C} \cdot y_{1}$. Now by an easy calculation, we find that $\mathrm{E}=\left.\mathcal{E}_{2}\right|_{\mathbb{P r} \times\{y\}}$ 
is the extension sheaf

$$
0 \rightarrow \mathcal{O}_{\mathrm{L}}(-1) \oplus \mathcal{O}_{\mathrm{L}}(-1) \rightarrow \mathrm{E} \rightarrow \mathcal{O}_{\mathrm{L}} \rightarrow 0
$$

determined by $y_{1}$ and the image of the line $y$ in $\operatorname{Ext}^{1}\left(\mathcal{O}_{L}, \mathcal{O}_{L}(-1)\right)$. Hence $E$ is stable if and only if $y \in \mathbb{P}\left(\operatorname{Ext}^{1}\left(\mathcal{O}_{\mathrm{L}}, \mathcal{O}_{\mathrm{L}}(-1)\right) \oplus \mathbb{C}\right)$ does not belong to the projective line spanned by $\mathrm{y}_{1}$ and the smoothing direction $\mathbb{C}$, in which case $\mathrm{E}=$ $\mathrm{F} \oplus \mathcal{O}_{\mathrm{L}}(-1)$ for a stable sheaf $\mathrm{F}$ with Hilbert polynomial $2 \mathrm{~m}+1$. The projective line spanned by $y_{1}$ and $\mathbb{C}$ is precisely $\Theta_{2}^{3} \cap q_{2}^{-1}\left(y_{1}\right)$.

Example 4.11. In fact, we can calculate all the stable sheaves in $\mathcal{E}_{2}$ by local calculations. Let $C$ be the union of two curves $\{(t, s) \mid t s=0\}$ and consider the family of stable maps locally given by

$$
x_{1}=t^{2}, \quad x_{2}=s, \quad x_{3}=a t, \quad a=t s .
$$

When $a \neq 0$, it is a family of smooth cubics and when $a=0$, we have a stable map in $\Theta^{2}$. Then on the line of $a \in \mathbb{C}$,

$$
\mathcal{E}_{0}=\mathcal{E}_{1}=\mathbb{C}[t, s]=\mathbb{C}[t, s, a] /(t s-a)
$$

and $\mathcal{E}_{2}$ is the kernel of

$$
\mathbb{C}[t, s] \longrightarrow \mathbb{C}[t, s] /(t s) \longrightarrow t \mathbb{C}\left[t^{2}\right]
$$

The central fiber of $\mathcal{E}_{2}$ is then

$$
\mathcal{E}_{2} / a \mathcal{E}_{2}=\mathbb{C}\left[\mathrm{t}^{2}, \mathrm{~s}\right] /\left(\mathrm{t}^{2} \mathrm{~s}^{2}\right) \cong \mathbb{C}\left[x_{1}, \cdots, x_{\mathrm{r}}\right] /\left(x_{1} x_{2}^{2}, x_{3}^{2}, x_{2} x_{3}^{2}, x_{3}-x_{1} x_{2}, x_{4}, \cdots, x_{r}\right) .
$$

This is a Cohen-Macaulay curve and hence stable. Of course, this is a curve with two components, one of which is a reduced line and the other is the double line thickened in a quadric surface in a $\mathbb{P}^{3}$.

Remark 4.12. We will see in the subsequent section that $\Gamma^{2}$ is a $\mathbb{P}_{(1,2,2)}^{2}$-bundle over a $\mathbb{P}^{r-1} \times \mathbb{P}^{r-1}$-bundle over $\mathbb{P}^{r}$ by GIT, where $\mathbb{P}_{(1,2,2)}^{2}$ is the weighted projective space with weights $(1,2,2)$. Of course, the $\mathbb{P}^{r-1} \times \mathbb{P}^{r-1}$-bundle over $\mathbb{P}^{r}$ above parameterizes pairs of intersecting lines in $\mathbb{P}^{r}$ and $\mathbb{P}_{(1,2,2)}^{2}$ parameterizes double coverings of a line. The proof of Lemma 4.10 tells us that the family $\mathcal{E}_{2}$ of stable sheaves on $\Gamma_{2}^{2}-\Gamma_{2}^{1} \cup \Gamma_{2}^{3}$ remains constant on the fibers $\mathbb{P}_{(1,2,2)}^{2}$ but depends only on the fiber $\mathbb{P} \operatorname{Ext}^{1}\left(\mathcal{O}_{\mathrm{C}^{\prime}}, \mathcal{O}_{\mathrm{L}_{2}}(-1)\right)$ of $\Gamma_{2}^{2} \rightarrow \Gamma_{1}^{2}$.

Let $\mathrm{q}_{3}: \mathrm{Q}_{3} \rightarrow \mathrm{Q}_{2}$ be the blow-up along $\Theta_{2}^{3}$ and let $\mathrm{p}_{3}: \mathbf{M}_{3} \rightarrow \mathbf{M}_{2}$ be its quotient by $\operatorname{SL}(2)$. Let $\Theta_{3}^{3}$ be the exceptional divisor and $\Theta_{3}^{1}, \Theta_{3}^{2}$ be the proper transforms of $\Theta_{2}^{1}, \Theta_{2}^{2}$ respectively. Let $\Gamma_{3}^{i}$ be the quotient of $\Theta_{3}^{i}$ by $\operatorname{SL}(2)$. Let $\mathcal{E}_{3}^{\prime}$ be the pull-back of $\mathcal{E}_{2}$ to $\mathbb{P}^{\mathrm{r}} \times \mathrm{Q}_{3}$. As we saw above, if $\mathrm{y} \in \Theta_{3}^{3}, \mathcal{E}_{3}^{\prime} \mid \mathbb{P r}^{\mathrm{r}} \times\{\mathrm{y}\} \cong \mathrm{F} \oplus \mathcal{O}_{\mathrm{L}}(-1)$ for some line $L$ and a stable sheaf $F$ whose Hilbert polynomial is $2 m+1$. Obviously, $\mathrm{F}$ is the destabilizing sheaf and by the existence of relative Quot scheme [9, Chapter 2 ] again, we obtain a quotient homomorphism

$$
\left.\mathcal{E}_{3}^{\prime}\right|_{\mathbb{P r} \times \Theta_{3}^{3}} \rightarrow A_{3}
$$

such that for $y \in \Theta_{3}^{3},\left.A_{3}\right|_{\mathbb{P r}^{r} \times\{y\}} \cong \mathcal{O}_{\mathrm{L}}(-1)$ for some line $\mathrm{L}$ depending on $\mathrm{y}$. Let $\mathcal{E}_{3}$ be the kernel of the epimorphism

$$
\left.\mathcal{E}_{3}^{\prime} \rightarrow \mathcal{E}_{3}^{\prime}\right|_{\mathbb{P r}^{r} \times \Theta_{3}^{3}} \rightarrow A_{3} .
$$


Lemma 4.13. $\mathcal{E}_{3}$ is a family of stable sheaves on $\mathbb{P}^{r}$ and hence we obtain an invariant morphism $\Psi_{3}: \mathrm{Q}_{3} \rightarrow \mathbf{S}$ which induces a morphism

$$
\Phi_{3}: \mathbf{M}_{3} \rightarrow \mathbf{S} .
$$

Proof. Let $y \in \Theta_{3}^{3}, y_{2}=q_{3}(y), y_{1}=q_{2}\left(y_{2}\right)$. From the proofs of Lemmas 4.6 and 4.10 , we find that the normal space to $\Theta_{2}^{3}$ in $Q_{2}$ at $y_{2}$ is

$$
\mathrm{N}_{\Theta_{2}^{3} / \mathrm{Q}_{2}, \mathrm{y}_{2}} \cong \operatorname{Ext}^{1}\left(\mathcal{O}_{\mathrm{L}}, \mathcal{O}_{\mathrm{L}}(-1)\right) / \mathbb{C} \cdot \mathrm{y}_{1} \oplus \mathbb{C} \cong \mathbb{C}^{\mathrm{r}-1}
$$

where the summand $\operatorname{Ext}^{1}\left(\mathcal{O}_{\mathrm{L}}, \mathcal{O}_{\mathrm{L}}(-1)\right) / \mathbb{C} \cdot \mathrm{y}_{1}$ is the normal space of $\Theta_{2}^{3}$ in $\Theta_{2}^{1}$ while the direct summand $\mathbb{C}$ is the normal direction to the divisor $\Theta_{2}^{1}$. The family $\mathcal{E}_{2}$ restricted to $\Theta_{2}^{3}$ is always of the splitting form $F \oplus \mathcal{O}_{\mathrm{L}}(-1)$ where $F$ is the extension of $\mathcal{O}_{\mathrm{L}}$ by $\mathcal{O}_{\mathrm{L}}(-1)$, i.e. the double line in a plane containing L. Hence the KodairaSpencer map

$$
\mathrm{T}_{\mathrm{y}_{2}} \mathrm{Q}_{2} \longrightarrow \operatorname{Ext}_{\mathbb{P r}}^{1}\left(\mathrm{~F} \oplus \mathcal{O}_{\mathrm{L}}(-1), \mathrm{F} \oplus \mathcal{O}_{\mathrm{L}}(-1)\right)
$$

induces a map

$$
\mathrm{N}_{\Theta_{2}^{3} / \mathrm{Q}_{2}, y_{2}} \longrightarrow \operatorname{Ext}_{\mathbb{P r}^{r}}^{1}\left(\mathrm{~F}, \mathcal{O}_{\mathrm{L}}(-1)\right) \text {. }
$$

We claim this is injective and hence after elementary modification $\left.\mathcal{E}_{3}\right|_{\mathbb{P}^{r} \times\{y\}}$ becomes a nontrivial extension of $\mathrm{F}$ by $\mathcal{O}_{\mathrm{L}}(-1)$ which is obviously stable.

From the exactness of $0 \rightarrow \mathcal{O}_{\mathrm{L}}(-1) \rightarrow \mathrm{F} \rightarrow \mathcal{O}_{\mathrm{L}} \rightarrow 0$, we obtain an exact sequence (4.16)

$0 \rightarrow \operatorname{Ext}^{1}\left(\mathcal{O}_{\mathrm{L}}, \mathcal{O}_{\mathrm{L}}(-1)\right) / \mathbb{C} \cdot \mathrm{y}_{1} \rightarrow \operatorname{Ext}^{1}\left(\mathrm{~F}, \mathcal{O}_{\mathrm{L}}(-1)\right) \rightarrow \operatorname{Ext}^{1}\left(\mathcal{O}_{\mathrm{L}}(-1), \mathcal{O}_{\mathrm{L}}(-1)\right) \rightarrow 0$.

Suppose $y \in \mathbb{P} \operatorname{Ext}^{1}\left(\mathcal{O}_{\mathrm{L}}, \mathcal{O}_{\mathrm{L}}(-1)\right) / \mathbb{C} \cdot y_{1}$ and fix $\tilde{y} \in \operatorname{Ext}^{1}\left(\mathcal{O}_{\mathrm{L}}, \mathcal{O}_{\mathrm{L}}(-1)\right)$ representing $y$. Then from the proofs of Lemmas 4.6 and 4.10, we find that the direction of $y$ in $\mathrm{N}_{\Theta_{2}^{3} / \mathrm{Q}_{2}, \mathrm{y}_{2}}$ is given by the family of extensions

$$
0 \longrightarrow \mathcal{O}_{\mathrm{L}}(-1) \oplus \mathcal{O}_{\mathrm{L}}(-1) \longrightarrow \mathrm{E} \longrightarrow \mathcal{O}_{\mathrm{L}} \longrightarrow 0
$$

whose extension class is $\left(y_{1}, \epsilon \tilde{y}\right)$. By direct calculation, the elementary modification at $\epsilon=0$ gives us the nontrivial extension with extension class $\left(y_{1}, \tilde{y}\right)$. This is just the thickening of $\mathrm{L}$ in a $\mathbb{P}^{3}$ determined by $\mathrm{y}_{1}$ and $\mathrm{y}$. Therefore, we find that the Kodaira-Spencer map sends the summand $\operatorname{Ext}^{1}\left(\mathcal{O}_{\mathrm{L}}, \mathcal{O}_{\mathrm{L}}(-1)\right) / \mathbb{C} \cdot \mathrm{y}_{1}$ of $\mathrm{N}_{\Theta_{2}^{3} / \mathrm{Q}_{2}, \mathrm{y}_{2}}$ isomorphically onto the same subspace $\operatorname{Ext}^{1}\left(\mathcal{O}_{\mathrm{L}}, \mathcal{O}_{\mathrm{L}}(-1)\right) / \mathbb{C} \cdot \mathrm{y}_{1}$ of $\operatorname{Ext}^{1}\left(\mathrm{~F}, \mathcal{O}_{\mathrm{L}}(-1)\right)$.

For the other summand $\mathbb{C}$ of $\mathrm{N}_{\Theta_{2}^{3} / \mathrm{Q}_{2}, y_{2}}$, consider the locus $\Lambda$ of stable maps in $Q_{0}$ whose images are planar. Then by direct local calculation, it is easy to see that this locus is of codimension $r-2$ and its proper transform $\Lambda_{2}$ in $Q_{2}$ intersects with $\Theta_{2}^{1}$ transversely along $\Theta_{2}^{3}$. We choose an analytic curve $g: D \rightarrow \Lambda_{2}$ from a small disk $D$ in $\mathbb{C}$ to $\Lambda_{2}$, which passes through $y_{2}$ and moves away from $\Theta_{2}^{1}$. Let $\mathcal{E}_{\mathrm{g}}$ be the pullback of $\mathcal{E}_{2}$ to $\mathbb{P}^{r} \times \mathrm{D}$ via $\left(\mathrm{id}_{\mathbb{P}}, \mathrm{g}\right)$. For $\mathrm{t} \neq 0$, the image of the stable map parameterized by $\mathrm{q}_{1}\left(\mathrm{q}_{2}(\mathrm{~g}(\mathrm{t}))\right)$ is a singular cubic plane curve $\mathrm{C}_{\mathrm{t}}^{\prime}$ and $\mathcal{E}_{2}$ at $t$ is an extension of a skyscraper sheaf $\mathcal{O}_{p_{t}}$ by the structure sheaf $\mathcal{O}_{C_{t}^{\prime}}$ of $C_{t}^{\prime}$ for some $p_{t} \in C_{t}^{\prime}$. Without loss of generality, by applying linear transformations, we may assume that $C_{t}^{\prime}$ are all contained in a fixed $\mathbb{P}^{2}$ in $\mathbb{P}^{r}$ for $t \neq 0$. The restriction of $\mathcal{E}_{\mathrm{g}}$ to $\mathrm{Spec} \mathbb{C}[\mathrm{t}] /\left(\mathrm{t}^{2}\right)$ is an extension

$$
0 \longrightarrow \mathrm{t} \cdot\left(\mathrm{F} \oplus \mathcal{O}_{\mathrm{L}}(-1)\right) \longrightarrow \mathcal{E}_{\mathrm{g}} \longrightarrow \mathrm{F} \oplus \mathcal{O}_{\mathrm{L}}(-1) \longrightarrow 0
$$

by flatness, where $F$ is the double of $L$ in $\mathbb{P}^{2}$. By the stability of $\mathcal{E}_{g}$ for $t \neq 0$, the extension class in $\operatorname{Ext}^{1}\left(\mathrm{~F} \oplus \mathcal{O}_{\mathrm{L}}(-1), \mathrm{F} \oplus \mathcal{O}_{\mathrm{L}}(-1)\right)$ has nontrivial component $\mathrm{c} \in$ 
$\operatorname{Ext}^{1}\left(\mathrm{~F}, \mathcal{O}_{\mathrm{L}}(-1)\right)$. Furthermore, since $\mathcal{E}_{\mathrm{g}}$ for $\mathrm{t} \neq 0$ is a family of stable sheaves supported in the plane $\mathbb{P}^{2}$ determined by $y_{1}$, if the image of $c$ in $\operatorname{Ext}^{1}\left(\mathcal{O}_{\mathrm{L}}(-1), \mathcal{O}_{\mathrm{L}}(-1)\right)$ by (4.16) were zero, then $c$ would come from $y_{1} \in \operatorname{Ext}^{1}\left(\mathcal{O}_{\mathrm{L}}, \mathcal{O}_{\mathrm{L}}(-1)\right)$ and hence $c$ would be trivial. Hence every sheaf in the family $\mathcal{E}_{3}$ is stable as desired.

There are three possibilities for $y \in \Theta_{3}^{3}$ and in each case one can calculate the sheaf $\mathcal{E}_{3}$ at the point as in the example below:

(1) If $y \in \mathbb{P} \operatorname{Ext}^{1}\left(\mathcal{O}_{\mathrm{L}}, \mathcal{O}_{\mathrm{L}}(-1)\right) / \mathbb{C}$, then $\left.\mathcal{E}_{3}\right|_{\mathbb{P r}^{r} \times\{y\}}$ is the thickening of $\mathrm{L}$ in a $\mathbb{P}^{3}$.

(2) If $y$ is the normal direction to $\Theta_{2}^{1}$, then $\mathcal{E}_{3}$ at $y$ is a nontrivial extension of a skyscraper sheaf $\mathbb{C}_{p}$ for some point $p \in L$ by the triple thickening of $L$ in a $\mathbb{P}^{2}$ which contains $\mathrm{L}$.

(3) If $y$ is not in either of the direct summands, then $\mathcal{E}_{3}$ at $y$ is a triple thickening of $L$ in a quadric cone in a $\mathbb{P}^{3}$ containing $L$.

As observed in Remark 4.8 and the above proof, the family of stable sheaves parameterized by $\Theta_{3}^{3}$ is independent of the factor $\left(\mathbb{P}^{7}\right)^{\text {s }}$, i.e. we may choose any stable map $y_{0}=q_{1}\left(y_{1}\right)$ to $L \cong \mathbb{P}^{1}$ in calculating the stable sheaves in $\mathcal{E}_{3}$.

Example 4.14. Case (2): Consider the family of maps $f_{a}: \mathbb{P}^{1} \rightarrow \mathbb{P}^{2}$, locally given by

$$
\mathbb{C}[x, y, a] \longrightarrow \mathbb{C}[t, a]=E
$$

where $x \mapsto a\left(\rho t+t^{2}\right), y \mapsto t^{3}, a \mapsto a$ for $\rho \in \mathbb{C}$. Before applying elementary modifications, we have

$$
\left.E\right|_{a=0}=\mathbb{C}[t]=\mathbb{C}[y] \oplus t \mathbb{C}[y] \oplus t^{2} \mathbb{C}[y] .
$$

The modification on $\mathrm{Q}_{1}$ is taking the kernel $\mathrm{E}_{1}$ of

$$
\mathbb{C}[t, a] \longrightarrow \mathbb{C}[t, a] /(a) \cong \mathbb{C}[t] \longrightarrow t \mathbb{C}[y] \oplus t^{2} \mathbb{C}[y] .
$$

Hence $E_{1}$ consists of elements of the form $f\left(t^{3}\right)+a \cdot g(t, a)$ for polynomials $f, g$. To obtain $\left.E_{1}\right|_{a=0}$, we have to take the quotient by the submodule generated by $a$. It is easy to see that the quotient by $(a)$ is

$$
\mathbb{C}[y] \oplus a t \mathbb{C}[y] \oplus a t^{2} \mathbb{C}[y] \cong \mathbb{C}[y] \oplus a t \mathbb{C}[y] \oplus x \mathbb{C}[y] .
$$

The modification on $\mathrm{Q}_{3}$ is taking the kernel $\mathrm{E}_{3}$ of

$$
\left.\mathrm{E}_{1} \rightarrow \mathrm{E}_{1}\right|_{\mathrm{a}=0} \cong \mathbb{C}[y] \oplus a t \mathbb{C}[y] \oplus x \mathbb{C}[y] \longrightarrow a t \mathbb{C}[y]
$$

If we quotient out $E_{3}$ by the submodule generated by $a$, we obtain

$$
\mathbb{C}[y] \oplus x \mathbb{C}[y] \oplus x^{2} \mathbb{C}[y]+\mathbb{C} \cdot a^{2} t=\mathbb{C}[x, y] /\left(x^{3}\right)+\mathbb{C}_{\left(0, \rho^{3}\right)}
$$

which is a nontrivial extension of $\mathbb{C}_{p}$ where $p=\left(0, \rho^{3}\right)$ by $\mathbb{C}[x, y] /\left(x^{3}\right)$, the triple thickening of the line $x=0$ in the $x y$-plane, because $x^{2}=a^{2} t\left(y-\rho^{3}\right)$ in $E_{3} / a E_{3}$.

Example 4.15. Case (3): Consider the family of maps $f_{a}: \mathbb{P}^{1} \rightarrow \mathbb{P}^{3}$, locally given by

$$
\mathbb{C}[x, y, z, a] \longrightarrow \mathbb{C}[t, a]=E
$$

where $x \mapsto a t^{2}, y \mapsto a^{2} t, z \mapsto t^{3}, a \mapsto a$. As above, $\left.E\right|_{a=0}=\mathbb{C}[t]=\mathbb{C}[z] \oplus t \mathbb{C}[z] \oplus$ $\mathrm{t}^{2} \mathbb{C}[z]$. The calculations as above show that

$$
\mathcal{E}_{3} / a \mathcal{E}_{3}=\mathbb{C}[z] \oplus x \mathbb{C}[z] \oplus y \mathbb{C}[z]
$$

and that $x y=0, y^{2}=0, y z-x^{2}=0$. This is the thickening of $L$ in the quadric cone $y z=x^{2}$. 
4.4. Blow-downs. In this subsection, we show that $\Phi_{3}: \mathbf{M}_{3} \rightarrow \mathbf{S}$ factors through three blow-downs

$$
\mathbf{M}_{3} \stackrel{\pi_{4}}{\longrightarrow} \mathbf{M}_{4} \stackrel{\pi_{5}}{\longrightarrow} \mathbf{M}_{5} \stackrel{\pi_{6}}{\longrightarrow} \mathbf{M}_{6}
$$

where $\pi_{4}$ is a weighted blow-down along $\Gamma_{3}^{2}, \pi_{5}$ is a weighted blow-down along the proper transform of $\Gamma_{3}^{3}$ and $\pi_{6}$ is the smooth blow-down along the proper transform of $\Gamma_{3}^{1}$. We will study the local geometry of the divisors and show that the divisor to be contracted at each stage is a (weighted) projective bundle and there exists a blow-down map contracting the projective fibers. Then it is easy to see that the morphism $\Phi_{3}$ remains constant on each contracted fiber and hence it factors through $\Phi_{6}: \mathbf{M}_{6} \rightarrow \mathbf{S}$. Finally one can directly check that the induced morphism

$$
\Phi_{6}: \mathbf{M}_{6} \longrightarrow \mathbf{S}
$$

is bijective and hence we obtain an isomorphism $\mathbf{M}_{6} \cong \mathbf{S}$.

Let us start with $\Gamma^{1}$, which is isomorphic to $\mathcal{M}_{0}(\mathbb{P U})$ over $\operatorname{Gr}(2, r+1)$. Recall that

$$
\Gamma^{1}=\Theta^{1} / \operatorname{SL}(2)
$$

where $\Theta^{1}$ is given by (4.2). Since all the transformations will take place over $\operatorname{Gr}(2, r+1)$, we fix a line $L \cong \mathbb{P}^{1} \subset \mathbb{P}^{r}$ to simplify the notation. In particular, $\Theta^{1}$ is a $\mathbb{P}\left(\operatorname{Sym}^{3}\left(\mathbb{C}^{2}\right) \otimes \mathbb{C}^{2}\right)^{s}$-bundle on $\operatorname{Gr}(2, r+1)$. By Corollary 4.3, the normal bundle of $\Theta^{1}$, restricted to a fiber of $\Theta^{1} \rightarrow \operatorname{Gr}(2, r+1)$ over $L$, is

$$
\mathcal{O}_{\mathbb{P}^{7}}(-1)^{2 r-2}=\operatorname{Ext}^{1}\left(\mathcal{O}_{\mathrm{L}}, \mathcal{O}_{\mathrm{L}}(-1)\right)^{\oplus 2} \otimes \mathcal{O}(-1)
$$

and hence an analytic neighborhood $\mathrm{U}^{1}$ of $\Gamma^{1}$ in $\mathbf{M}$ is equivalent to a bundle over $\operatorname{Gr}(2, r+1)$ with fiber

$$
\tilde{\mathrm{U}}^{1}:=\mathcal{O}_{\mathbb{P}^{7}}(-1)^{2 \mathrm{r}-2} / / \mathrm{SL}(2) .
$$

Therefore, a fiber of $\Gamma_{1}^{1} \rightarrow \operatorname{Gr}(2, r+1)$ over $\mathrm{L}$ is

$$
\tilde{\Gamma}_{1}^{1}:=\left(\mathbb{P}\left(\operatorname{Sym}^{3}\left(\mathbb{C}^{2}\right) \otimes \mathbb{C}^{2}\right) \times \mathbb{P}\left(\mathbb{C}^{r-1} \otimes \mathbb{C}^{2}\right)\right) / / \mathcal{O}(1, \lambda) \operatorname{SL}(2)
$$

which is the GIT quotient of $\mathbb{P}^{7} \times \mathbb{P}^{2 r-3}$ with linearization $\mathcal{O}(1, \lambda)=\mathcal{O}_{\mathbb{P}^{7}}(1) \otimes$ $\left.\mathcal{O}_{\mathbb{P}^{2 r-3}}(\lambda)\right)$ for $0<\lambda<1$. (Note that for $\lambda<1$, the stable set is $\left(\mathbb{P}^{7}\right)^{s} \times \mathbb{P}^{2 r-3}$.) Here SL(2) acts trivially on $\mathbb{C}^{r-1}$ and as standard matrix multiplication on $\mathbb{C}^{2}$ for $\mathbb{P}\left(\mathbb{C}^{r-1} \otimes \mathbb{C}^{2}\right)$. Furthermore, an analytic neighborhood $\mathrm{U}_{1}^{1}$ of $\Gamma_{1}^{1}$ is the quotient of the line bundle

$$
\tilde{\mathrm{u}}_{1}^{1}:=\mathcal{O}_{\mathbb{P}^{7} \times \mathbb{P}^{2 r-3}}(-1,-1) / / \mathcal{O}(1, \lambda) \operatorname{SL}(2)
$$

since the blow-up of $\mathcal{O}_{\mathbb{P}^{7}}(-1)^{2 r-2}$ along the zero section is $\mathcal{O}_{\mathbb{P}^{7} \times \mathbb{P}^{2 r-3}}(-1,-1)$. Here

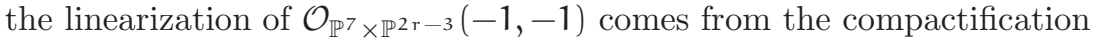

$$
\mathbb{P}\left(\mathcal{O}_{\mathbb{P}^{7} \times \mathbb{P}^{2 r-3}}(-1,-1) \oplus \mathcal{O}\right)
$$

with linearization

$$
\pi^{*} \mathcal{O}_{\mathbb{P}^{7} \times \mathbb{P}^{2 r-3}}(1, \lambda) \otimes \mathcal{O}_{\mathbb{P}(\mathcal{O}(-1,-1) \oplus \mathcal{O})}(-\epsilon Z)
$$

where $\mathbf{Z} \cong \mathbb{P}^{7} \times \mathbb{P}^{2 r-3}$ is the zero section $\mathbb{P}(0 \oplus \mathcal{O})$ of

$$
\pi: \mathbb{P}\left(\mathcal{O}_{\mathbb{P}^{7}} \times \mathbb{P}^{2 r-3}(-1,-1) \oplus \mathcal{O}\right) \rightarrow \mathbb{P}^{7} \times \mathbb{P}^{2 r-3}
$$

and $\epsilon$ is a sufficiently small positive number.

When $\lambda>>0$, the quotient

$$
\tilde{\mathrm{U}}_{5}^{1}:=\mathcal{O}_{\mathbb{P}^{7} \times \mathbb{P}^{2 r-3}}(-1,-1) / / \mathcal{O}(1, \lambda) \operatorname{SL}(2)
$$


is the blow-up of

$$
\tilde{\mathrm{U}}_{6}^{1}:=\mathcal{O}_{\mathbb{P}^{2 r-3}}(-1)^{8} / / \mathrm{SL}(2)
$$

along the zero section

$$
\tilde{\Gamma}_{6}^{1}:=\mathbb{P}^{2 r-3} / / \operatorname{SL}(2) \cong \operatorname{Gr}(2, r-1)
$$

and we have a blow-down map $\tilde{\mathrm{U}}_{5}^{1} \longrightarrow \tilde{\mathrm{U}}_{6}^{1}$ to a neighborhood of $\tilde{\Gamma}_{6}^{1}$. This Grassmannian $\tilde{\Gamma}_{6}^{1}$ parameterizes the choice of a $\mathbb{P}^{3}$ containing the line $\mathrm{L}$ and each point will give us a thickening of $\mathrm{L}$ in the chosen $\mathbb{P}^{3}$.

We will see that the blow-up process in the previous section can be described by variation of GIT quotients $[19,1]$, as we vary $\lambda$ from $1^{-}$to $\infty$. It is easy to see by the Hilbert-Mumford criterion that the quotient varies only at $\lambda=1$ and $\lambda=3$. Then one finds the $\mathbb{C}^{*}$-fixed locus and the weight space decomposition of the normal bundle to each fixed point component. It is just an elementary exercise to show that the variations at $\lambda=1$ and $\lambda=3$ are flips, i.e. a blow-up followed by a blow-down, as follows.

(1) The flip at $\lambda=1$ :

$$
\tilde{\Gamma}_{1}^{1}=\mathbb{P}^{7} \times \mathbb{P}^{2 r-3} / / \mathcal{O}(1,1-) \operatorname{SL}(2) \longleftarrow \tilde{\Gamma}_{2}^{1} \longrightarrow \mathbb{P}^{7} \times \mathbb{P}^{2 r-3} / / \mathcal{O}(1,2) \operatorname{SL}(2)
$$

is the composition of a blow-up and a blow-down. The first map is the smooth blow-up along a $\mathbb{P}_{(1,2,2)}^{2}$-bundle over $\mathbb{P}^{1} \times \mathbb{P}^{r-2}$, where $\mathbb{P}_{(1,2,2)}^{2}$ is the weighted projective space with weights $(1,2,2)$. This blow-up center coincides with the fiber of the intersection $\Gamma_{1}^{1} \cap \Gamma_{1}^{2}$ over $\operatorname{Gr}(2, r+1)$. The second map is the weighted blow-up along a $\mathbb{P}^{r-1}$-bundle over $\mathbb{P}^{1} \times \mathbb{P}^{r-2}$ with weights $(1,2,2)$ on each normal space.

(2) The flip at $\lambda=3$ :

$$
\mathbb{P}^{7} \times \mathbb{P}^{2 r-3} / / \mathcal{O}(1,2) \operatorname{SL}(2) \longleftarrow \tilde{\Gamma}_{4}^{1} \longrightarrow \mathbb{P}^{7} \times \mathbb{P}^{2 r-3} / / \mathcal{O}(1,4) \operatorname{SL}(2)=: \tilde{\Gamma}_{5}^{1}
$$

is the composition of a blow-up and a blow-down. The first map is the smooth blow-up along a $\mathbb{P}_{(1,2,2,3,3)}^{4}$-bundle over $\mathbb{P}^{1} \times \mathbb{P}^{\text {r- }-2}$ where $\mathbb{P}_{(1,2,2,3,3)}^{4}$ is the weighted projective space with weights $(1,2,2,3,3)$. The second map is the weighted blow-up along a $\mathbb{P}^{r-3}$-bundle over $\mathbb{P}^{1} \times \mathbb{P}^{r-2}$ with weights $(1,2,2,3,3)$ on each normal space.

When $\lambda>3, \tilde{\Gamma}_{5}^{1}=\mathbb{P}^{7} \times \mathbb{P}^{2 r-3} / / \mathcal{O}(1, \lambda) \operatorname{SL}(2)$ is a $\mathbb{P}^{7}$-bundle over

$$
\mathbb{P}\left(\mathbb{C}^{r-1} \otimes \mathbb{C}^{2}\right) / / \operatorname{SL}(2) \cong \mathrm{Gr}(2, \mathrm{r}-1)=: \tilde{\Gamma}_{6}^{1} .
$$

If we let $\tilde{\Gamma}_{3}^{1}$ be the fiber product of $\tilde{\Gamma}_{2}^{1}$ with $\tilde{\Gamma}_{4}^{1}$, we obtain the following diagram.

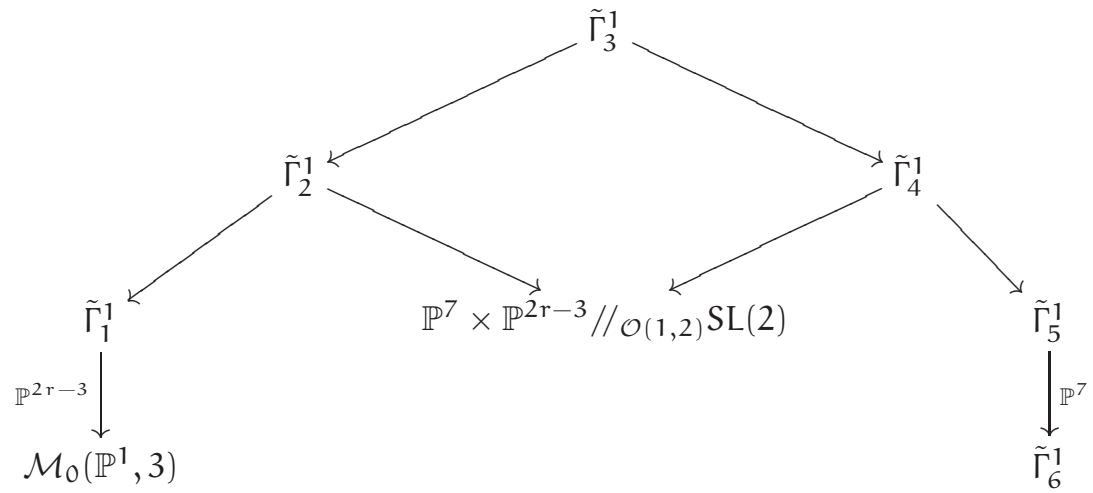


The left and right vertical maps are projective bundles and all other maps are blow-ups. The two maps from $\tilde{\Gamma}_{3}^{1}$ are blow-ups because the blow-up centers in $\mathbb{P}^{7} \times \mathbb{P}^{2 r-3} / / \mathcal{O}_{(1,2)} \operatorname{SL}(2)$ are transversal. By our construction, it is easy to see that $\Gamma_{i}^{1}$ is exactly a $\tilde{\Gamma}_{i}^{1}$-bundle over $\operatorname{Gr}(2, r+1)$ for $i \leq 3$.

Similarly, we can study the variation of the GIT quotient

$$
\tilde{\mathrm{U}}_{1}^{1}=\mathcal{O}_{\mathbb{P}^{7} \times \mathbb{P}^{2 r-3}}(-1,-1) / / \operatorname{SL}(2)
$$

with linearization

$$
\pi^{*} \mathcal{O}_{\mathbb{P}^{7} \times \mathbb{P}^{2 r-3}}(1, \lambda) \otimes \mathcal{O}_{\mathcal{O}(-1,1)}(-\epsilon Z)
$$

where $Z \cong \mathbb{P}^{7} \times \mathbb{P}^{2 r-3}$ is the zero section as we vary $\lambda$ from $1^{-}$to $\infty$. As above, the GIT quotient varies only at $\lambda=1$ and $\lambda=3$.

(1) The wall crossing at $\lambda=1$ takes place over the $\mathbb{C}^{*}$-fixed point component $\mathrm{B}_{1}$, which is the restriction of the line bundle $\mathcal{O}(-1,-1)$ to the flip base $\mathbb{P}^{1} \times \mathbb{P}^{r-2}$ for $\tilde{\Gamma}^{1}$ at $\lambda=1$ above. The flip at $\lambda=1$ is the composition

$$
\tilde{\mathrm{U}}_{1}^{1}=\mathcal{O}_{\mathbb{P}^{7} \times \mathbb{P}^{2 r-3}}(-1,-1) / / \lambda=1-\mathrm{SL}(2) \longleftarrow \tilde{\mathrm{U}}_{2}^{1} \longrightarrow \mathcal{O}_{\mathbb{P}^{7} \times \mathbb{P}^{2 r-3}}(-1,-1) / / \lambda_{=2} \operatorname{SL}(2)
$$

of the smooth blow-up along a $\mathbb{P}_{(1,2,2)}^{2}$-bundle over $B_{1}$ and the weighted blow-up along a $\mathbb{P}^{r-1}$-bundle over $\mathrm{B}_{1}$.

(2) The wall crossing at $\lambda=3$ takes place over the $\mathbb{C}^{*}$-fixed component $\mathrm{B}_{2} \cong$ $\mathbb{P}^{1} \times \mathbb{P}^{r-2}$, which is the zero section of the restriction of the line bundle $\mathcal{O}(-1,-1)$ to the flip base $\mathbb{P}^{1} \times \mathbb{P}^{r-2}$ for $\tilde{\Gamma}^{1}$ at $\lambda=3$ above. The flip at $\lambda=3$ is the composition

$$
\mathcal{O}_{\mathbb{P}^{7} \times \mathbb{P}^{2 r-3}}(-1,-1) / / \lambda_{=2} \operatorname{SL}(2) \longleftarrow \tilde{\mathrm{U}}_{4}^{1} \longrightarrow \mathcal{O}_{\mathbb{P}^{7} \times \mathbb{P}^{2 r-3}}(-1,-1) / / \lambda=4 \operatorname{SL}(2)=\tilde{\mathrm{U}}_{5}^{1}
$$

is the composition of the smooth blow-up along a $\mathbb{P}_{(1,2,2,3,3)}^{4}$-bundle over $\mathrm{B}_{2}$ and the weighted blow-up along a $\mathbb{P}^{\mathrm{r}-2}$-bundle over $\mathrm{B}_{2}$ with weights $(1,2,2,3,3)$ on each normal space.

In summary, we have the following diagram of blow-ups for $\tilde{u}_{j}^{1}$ :

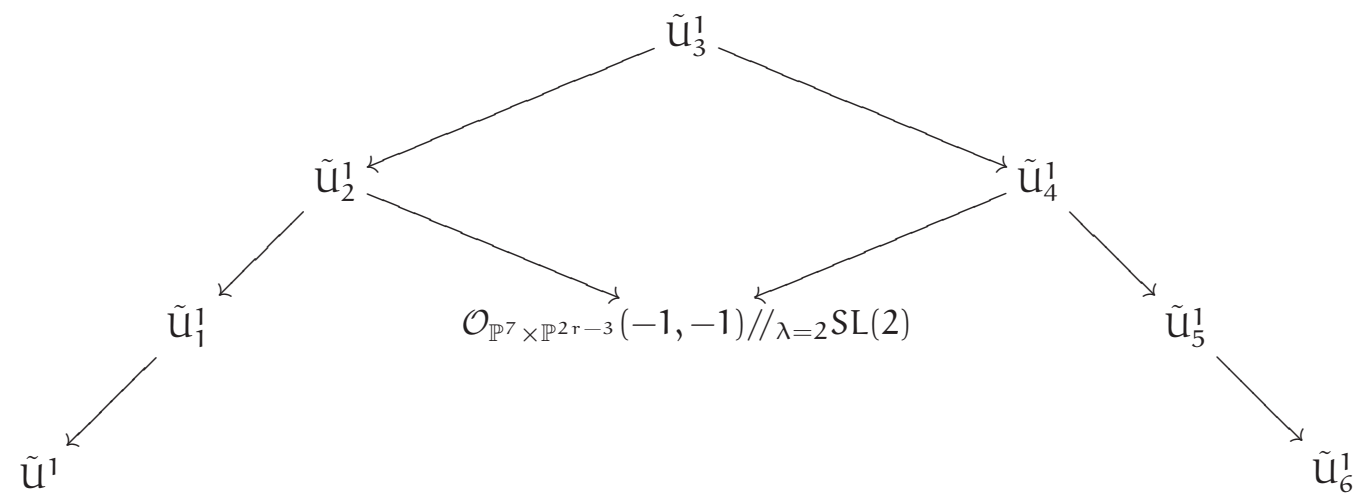

Here $\tilde{\mathrm{U}}_{3}^{1}$ is the fiber product of $\tilde{\mathrm{U}}_{2}^{1}$ and $\tilde{\mathrm{U}}_{4}^{1}$ over $\mathcal{O}_{\mathbb{P}^{7} \times \mathbb{P}^{2 r-3}(-1,-1) / / \lambda_{=2}} \mathrm{SL}(2)$. As

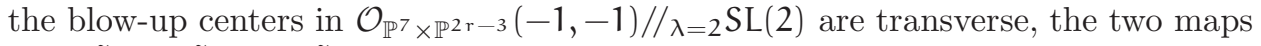
from $\tilde{\mathrm{U}}_{3}^{1}$ to $\tilde{\mathrm{U}}_{2}^{1}$ and $\tilde{\mathrm{U}}_{4}^{1}$ are blow-ups.

Let $\mathrm{u}_{j}^{1} \subset \mathbf{M}_{j}, j=1,2,3$ be the inverse image of the neighborhood $\mathrm{U}^{1}$ of $\Gamma^{1}$. Here $\mathrm{U}^{1}$ is a bundle over $\operatorname{Gr}(2, r+1)$ with fiber $\tilde{U}^{1}$. From our construction, it is 
easy to check that the restrictions of the blow-ups

$$
\mathrm{M}_{3} \longrightarrow \mathrm{M}_{2} \longrightarrow \mathrm{M}_{1} \longrightarrow \mathrm{M}
$$

to $\mathrm{U}^{1}$ coincide with the three blow-ups on the left side of (4.19). If we can check that the first blow-down $\tilde{\mathrm{U}}_{3}^{1} \rightarrow \tilde{\mathrm{U}}_{4}^{1}$ is extended to a global contraction map $\mathrm{p}_{4}$ : $\mathbf{M}_{3} \rightarrow \mathbf{M}_{4}$ along $\Gamma_{3}^{2}$, then simply by gluing with $\mathbf{M}_{4}-\Gamma_{4}^{1} \cup \Gamma_{4}^{3}$ where $\Gamma_{4}^{i}=p_{4}\left(\Gamma_{3}^{i}\right)$, the blow-downs

$$
\tilde{\mathrm{U}}_{4}^{1} \longrightarrow \tilde{\mathrm{U}}_{5}^{1} \longrightarrow \tilde{\mathrm{U}}_{6}^{1}
$$

extends to a global maps

$$
\mathrm{M}_{4} \longrightarrow \mathrm{M}_{5} \longrightarrow \mathrm{M}_{6}
$$

which contracts along the proper transform $\Gamma_{4}^{3}$ of $\Gamma_{3}^{3}$ and then along the proper transform $\Gamma_{5}^{1}$ of $\Gamma_{3}^{1}$, because $\Gamma_{j}^{1}$ and $\Gamma_{j}^{3}$ are both contained in $\mathrm{u}_{j}^{1}$.

So let us now study the blow-ups of $\Gamma^{2}$. Recall that

$$
\Gamma^{2}=\Theta^{2} / \mathrm{SL}(2)
$$

where $\Theta^{2}$ is a $\mathbb{P}^{r-1}$-bundle over a bundle over $\operatorname{Gr}(2, r+1)$ with fiber

$$
\left[\mathbb{P}^{1} \times \mathbb{P}\left(\operatorname{Sym}^{2}\left(\mathbb{C}^{2}\right) \otimes \mathbb{C}^{2}\right)\right]^{s} \text {. }
$$

As before, we fix $L \in G r(2, r+1)$ to simplify our notation because everything takes place over $\operatorname{Gr}(2, r+1)$ in a neighborhood of $\Theta^{2}$. Consider the variation of the GIT quotient

$$
\mathbb{P}^{1} \times \mathbb{P}\left(\operatorname{Sym}^{2}\left(\mathbb{C}^{2}\right) \otimes \mathbb{C}^{2}\right) / / \mathcal{O}(1, \lambda) \operatorname{SL}(2)
$$

as we vary $\lambda$ from 1 to $0^{+}$. It is again an elementary exercise of GIT variation $([19,1])$ to see that the quotient varies only at $\lambda=1 / 2$ but there are no stable points if $\lambda<1 / 2$. The variation at $\lambda=1 / 2$ tells us that

$$
\mathbb{P}^{1} \times \mathbb{P}\left(\operatorname{Sym}^{2}\left(\mathbb{C}^{2}\right) \otimes \mathbb{C}^{2}\right) / / \mathcal{O}(1,1) \operatorname{SL}(2)
$$

is a $\mathbb{P}_{(1,2,2)}^{2}$-bundle over $\mathbb{P}^{1}=\mathrm{L}$. Since the $\mathbb{P}^{1}$-bundle over $\operatorname{Gr}(2, r+1)$ is a $\mathbb{P}^{r-1}$ bundle over $\mathbb{P}^{r}, \Gamma^{2}$ is a $\mathbb{P}_{(1,2,2)}^{2}$-bundle over a $\left(\mathbb{P}^{r-1} \times \mathbb{P}^{r-1}\right)$-bundle over $\mathbb{P}^{r}$. Obviously, the $\left(\mathbb{P}^{r-1} \times \mathbb{P}^{r-1}\right)$-bundle over $\mathbb{P}^{r}$ parameterizes (ordered) pairs of lines meeting at a point while $\mathbb{P}_{(1,2,2)}^{2}$ parameterizes double coverings for the first (or second) line.

We can easily keep track of $\Gamma^{2}$ through the blow-ups $p_{1}, p_{2}, p_{3}$. Firstly, $\Gamma_{1}^{2}$ is just the blow-up of $\left(\mathbb{P}^{r-1} \times \mathbb{P}^{r-1}\right)$ along the diagonal. Secondly, $\Gamma_{2}^{2}$ is a $\mathbb{P}^{r-1}$-bundle over $\Gamma_{1}^{2}$. From the proof of Lemma 4.10, we see that the normal bundle to $\Theta_{1}^{2}$ is independent of the fiber $\mathbb{P}_{(1,2,2)}^{2}$ and hence $\Gamma_{2}^{2}$ is in fact a $\mathbb{P}_{(1,2,2)}^{2} \times \mathbb{P}^{r-1}$-bundle over a $b_{\mathbb{P}^{r-1}}\left(\mathbb{P}^{r-1} \times \mathbb{P}^{r-1}\right)$-bundle over $\mathbb{P}^{r}$. Finally $\Gamma_{3}^{2}$ replaces $\mathbb{P}_{(1,2,2)}^{2} \times \mathbb{P}^{r-1}$ by $\mathbb{P}_{(1,2,2)}^{2} \times \mathrm{bl}_{\mathbb{P}^{1}} \mathbb{P}^{\mathrm{r}-1}$. We claim that the $\mathbb{P}_{(1,2,2)}^{2}$ fibers of $\Gamma_{3}^{2}$ can be contracted to give us a map $\mathbf{M}_{3} \rightarrow \mathbf{M}_{4}$. Indeed, this claim easily follows from the following two observations. Firstly, from our description of the map $\tilde{\mathrm{U}}_{3}^{1} \rightarrow \tilde{\mathrm{U}}_{4}^{1}$, a neighborhood of $\mathrm{u}_{3}^{1} \cap \Gamma_{3}^{2}$ is a $\mathcal{O}_{\mathbb{P}_{(1,2,2)}^{2}}(-1)$-bundle over $\mathrm{U}_{3}^{1} \cap \Gamma_{3}^{2}$ and hence can be contracted to a $\mathbb{C}^{3}$ bundle. Secondly, any pair of distinct intersecting lines in $\mathbb{P}^{r}$ can be sent to another pair of distinct intersecting lines by the action of $\mathrm{GL}(r+1)$ and hence an analytic neighborhood of any point in $\Gamma_{3}^{2}$ is isomorphic to an analytic neighborhood for a point in $\mathrm{U}_{3}^{1} \cap \Gamma_{3}^{2}$. Since the action of an element in $G L(r+1)$ preserves the blowdown $\mathrm{U}_{3}^{1} \rightarrow \mathrm{U}_{4}^{1}$, we obtain the desired map $\mathrm{p}_{4}: \mathbf{M}_{3} \rightarrow \mathbf{M}_{4}$ which is a weighted 
blow-up along $\Gamma_{4}^{2}$. Therefore, we have analytic maps $\mathbf{M}_{3} \rightarrow \mathbf{M}_{4} \rightarrow \mathbf{M}_{5} \rightarrow \mathbf{M}_{6}$ contracting the divisors $\Gamma_{3}^{2}, \Gamma_{4}^{3}, \Gamma_{5}^{1}$ respectively.

Finally from our construction of the stable sheaves in the previous subsection, it is an easy but tedious exercise to check that set-theoretically the map $\Phi_{3}: \mathbf{M}_{3} \rightarrow \mathbf{S}$ factors through the maps $\mathbf{M}_{3} \rightarrow \mathbf{M}_{4} \rightarrow \mathbf{M}_{5} \rightarrow \mathbf{M}_{6}$ and hence we obtain an analytic map

$$
\Phi_{6}: \mathbf{M}_{6} \longrightarrow \mathbf{S} \text {. }
$$

Furthermore, it is easy to see that $\Phi_{6}$ is bijective. Therefore, we obtain the following by Zariski's main theorem.

Theorem 4.16. $\mathbf{S}$ is obtained from $\mathbf{M}$ by blowing up along $\Gamma^{1}, \Gamma_{1}^{2}, \Gamma_{2}^{3}$ and then blowing down along $\Gamma_{3}^{2}, \Gamma_{4}^{3}, \Gamma_{5}^{1}$.

The following diagram summarizes the results of this paper.

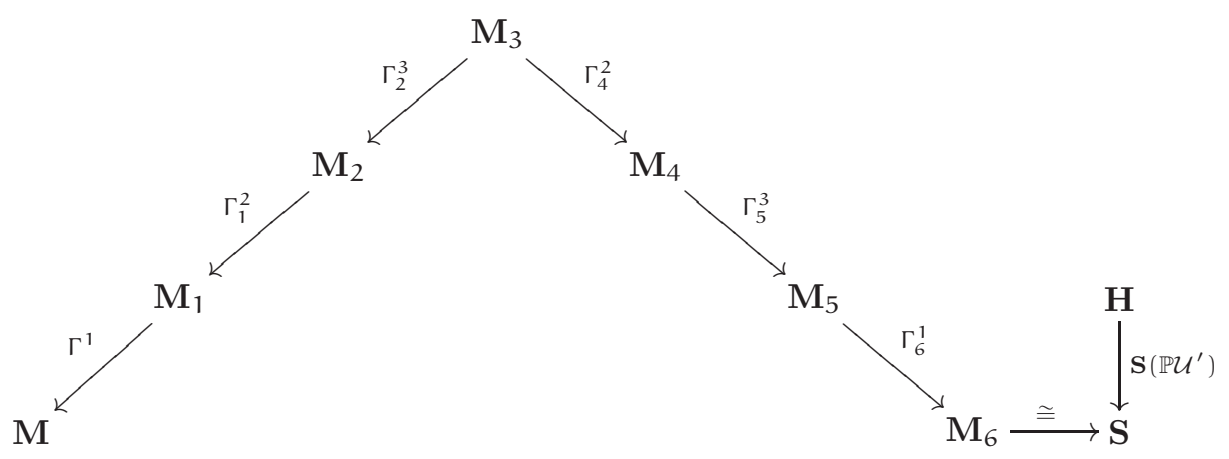

All the arrows are blow-ups and the blow-up centers are indicated above the arrows.

\section{Cohomology calculation}

We use Theorem 4.16 to calculate the Betti numbers of $\mathbf{S}$, by the blow-up formula [6]. We define the Poincaré polynomial of a topological space $X$ as

$$
P_{t}(X)=\sum_{i} t^{i} \operatorname{dim} H^{i}(X)
$$

From [11], the Poincaré polynomial of $\mathbf{M}=\mathcal{M}_{0}\left(\mathbb{P}^{r}, 3\right)$ is

$$
P_{t}(M)=\left(\frac{1-t^{2 r+10}}{1-t^{6}}+2 \frac{t^{4}-t^{2 r+4}}{1-t^{4}}\right) \frac{\left(1-t^{2 r+2}\right)}{\left(1-t^{2}\right)} \frac{\left(1-t^{2 r+2}\right)\left(1-t^{2 r}\right)}{\left(1-t^{2}\right)\left(1-t^{4}\right)} .
$$

The first blow-up adds

$$
P_{t}\left(\Gamma^{1}\right) \frac{t^{2}-t^{4 r-4}}{1-t^{2}}=\left(1+t^{2}+2 t^{4}+t^{6}+t^{8}\right) \frac{1-t^{2 r+2}}{1-t^{2}} \frac{1-t^{2 r}}{1-t^{4}} \frac{t^{2}-t^{4 r-4}}{1-t^{2}} .
$$

The second blow-up adds

$$
P_{t}\left(\Gamma_{1}^{2}\right) \frac{t^{2}-t^{2 r}}{1-t^{2}}=\left(1+t^{2}+t^{4}\right) \frac{1-t^{2 r}}{1-t^{2}}\left(\frac{1-t^{2 r}}{1-t^{2}}+\frac{t^{2}-t^{2 r-2}}{1-t^{2}}\right) \frac{1-t^{2 r+2}}{1-t^{2}} \frac{t^{2}-t^{2 r}}{1-t^{2}} .
$$

The third blow-up adds

$$
P_{t}\left(\Gamma_{2}^{3}\right) \frac{t^{2}-t^{2 r-2}}{1-t^{2}}=\left(\left(1+t^{2}\right)\left(1+t^{2}+2 t^{4}+t^{6}+t^{8}\right)+t^{2}\left(1+t^{2}\right)\left(1+t^{2}+t^{4}\right)\right)
$$




$$
\frac{1-t^{2 r-2}}{1-t^{2}} \frac{1-t^{2 r+2}}{1-t^{2}} \frac{1-t^{2 r}}{1-t^{4}} \frac{t^{2}-t^{2 r-2}}{1-t^{2}} .
$$

The first blow-down subtracts

$$
\begin{aligned}
P_{t}\left(\Gamma_{4}^{2}\right) \cdot\left(t^{2}+t^{4}\right)=\left[\frac{1-t^{2 r}}{1-t^{2}}\left(\frac{1-t^{2 r}}{1-t^{2}}+\frac{t^{2}-t^{2 r-2}}{1-t^{2}}\right)+\left(1+t^{2}\right) \frac{1-t^{2 r-2}}{1-t^{2}} \frac{t^{2}-t^{2 r-2}}{1-t^{2}}\right] \\
\\
\cdot \frac{1-t^{2 r}}{1-t^{2}} \frac{1-t^{2 r+2}}{1-t^{2}}\left(t^{2}+t^{4}\right) .
\end{aligned}
$$

The second blow-down subtracts

$$
P_{t}\left(\Gamma_{5}^{3}\right) \frac{t^{2}-t^{10}}{1-t^{2}}=\left(1+t^{2}\right)\left(\frac{1-t^{2 r-2}}{1-t^{2}}\right)^{2} \frac{1-t^{2 r+2}}{1-t^{2}} \frac{1-t^{2 r}}{1-t^{4}} \frac{t^{2}-t^{10}}{1-t^{2}}
$$

The third blow-down subtracts

$$
P_{t}\left(\Gamma_{6}^{1}\right) \frac{t^{2}-t^{16}}{1-t^{2}}=\frac{1-t^{2 r-2}}{1-t^{2}} \frac{1-t^{2 r-4}}{1-t^{4}} \frac{1-t^{2 r+2}}{1-t^{2}} \frac{1-t^{2 r}}{1-t^{4}} \frac{t^{2}-t^{16}}{1-t^{2}} .
$$

In summary, we obtain

Theorem 5.1. The Poincaré polynomial of $\mathbf{S}$ is

$$
\begin{aligned}
& \left(\frac{1-t^{2 r+10}}{1-t^{6}}+2 \frac{t^{4}-t^{2 r+4}}{1-t^{4}}\right) \frac{\left(1-t^{2 r+2}\right)}{\left(1-t^{2}\right)} \frac{\left(1-t^{2 r+2}\right)\left(1-t^{2 r}\right)}{\left(1-t^{2}\right)\left(1-t^{4}\right)} \\
& +\left(1+t^{2}+2 t^{4}+t^{6}+t^{8}\right) \frac{1-t^{2 r+2}}{1-t^{2}} \frac{1-t^{2 r}}{1-t^{4}} \frac{t^{2}-t^{4 r-4}}{1-t^{2}} \\
& +\left(1+t^{2}+t^{4}\right) \frac{1-t^{2 r}}{1-t^{2}}\left(\frac{1-t^{2 r}}{1-t^{2}}+\frac{t^{2}-t^{2 r-2}}{1-t^{2}}\right) \frac{1-t^{2 r+2}}{1-t^{2}} \frac{t^{2}-t^{2 r}}{1-t^{2}} \\
& +\left(\left(1+t^{2}\right)\left(1+t^{2}+2 t^{4}+t^{6}+t^{8}\right)+t^{2}\left(1+t^{2}\right)\left(1+t^{2}+t^{4}\right)\right) \\
& \frac{1-\mathrm{t}^{2 \mathrm{r}-2}}{1-\mathrm{t}^{2}} \frac{1-\mathrm{t}^{2 \mathrm{r}+2}}{1-\mathrm{t}^{2}} \frac{1-\mathrm{t}^{2 \mathrm{r}}}{1-\mathrm{t}^{4}} \frac{\mathrm{t}^{2}-\mathrm{t}^{2 \mathrm{r}-2}}{1-\mathrm{t}^{2}} \\
& -\left[\frac{1-t^{2 r}}{1-t^{2}}\left(\frac{1-t^{2 r}}{1-t^{2}}+\frac{t^{2}-t^{2 r-2}}{1-t^{2}}\right)+\left(1+t^{2}\right) \frac{1-t^{2 r-2}}{1-t^{2}} \frac{t^{2}-t^{2 r-2}}{1-t^{2}}\right] \\
& \frac{1-t^{2 r}}{1-t^{2}} \frac{1-t^{2 r+2}}{1-t^{2}}\left(t^{2}+t^{4}\right) \\
& -\left(1+t^{2}\right)\left(\frac{1-t^{2 r-2}}{1-t^{2}}\right)^{2} \frac{1-t^{2 r+2}}{1-t^{2}} \frac{1-t^{2 r}}{1-t^{4}} \frac{t^{2}-t^{10}}{1-t^{2}} \\
& -\frac{1-t^{2 r-2}}{1-t^{2}} \frac{1-t^{2 r-4}}{1-t^{4}} \frac{1-t^{2 r+2}}{1-t^{2}} \frac{1-t^{2 r}}{1-t^{4}} \frac{t^{2}-t^{16}}{1-t^{2}} \text {. }
\end{aligned}
$$

In particular, when $r=3$, we obtain

$P_{t}(\mathbf{H})=P_{t}(\mathbf{S})=1+2 t^{2}+6 t^{4}+10 t^{6}+16 t^{8}+19 t^{10}+22 t^{12}+19 t^{14}+16 t^{16}+10 t^{18}+6 t^{20}+2 t^{22}+t^{24}$

which coincides with the calculation in [2]. 


\section{REFERENCES}

1. I. Dolgachev and Y. Hu. Variation of geometric invariant theory quotients. Publ. IHES. 87 (1998), 5-56.

2. G. Ellingsrud, R. Piene and S. Strmme. On the variety of nets of quadrics defining twisted cubics. Space curves (Rocca di Papa, 1985), 84-96, Lecture Notes in Math., 1266, Springer, Berlin, 1987.

3. H. Freiermuth and G. Trautmann. On the moduli scheme of stable sheaves supported on cubic space curves. Amer. J. Math. 126 (2004), no. 2, 363-393.

4. W. Fulton. Intersection theory. Ergebnisse der Mathematik und ihrer Grenzgebiete (3), 2. Springer-Verlag, Berlin, 1984.

5. W. Fulton and R. Pandharipande. Notes on stable maps and quantum cohomology. Proceedings of Symposia in Pure Mathematics: Algebraic Geometry Santa Cruz 1995, J. Kollar, R. Lazarsfeld, D. Morrison Eds., Volume 62, Part 2, 45-96.

6. P. Griffiths and J. Harris. Principles of Algebraic Geometry. Wiley, New York, 1978.

7. J. Harris. Curves in Projective Space. Les Presses de lUniversite de Montreal: Montreal, 1982.

8. R. Hartshorne. Algebraic geometry. Graduate Texts in Mathematics, No. 52. Springer-Verlag, 1977.

9. D. Huybrechts and M. Lehn. The geometry of moduli spaces of sheaves. Aspects of Mathematics, E31. Friedr. Vieweg Sohn, Braunschweig, 1997.

10. Y.-H. Kiem. Hecke correspondence, stable maps and the Kirwan desingularization. Duke Math. J. 136 (2007) 585-618.

11. Y.-H. Kiem and H. Moon. Moduli space of stable maps to projective space via GIT. Preprint available at http://www.math.snu.ac.kr/ kiem

12. F.C. Kirwan, Cohomology of quotients in symplectic and algebraic geometry. Math. Notes vol. 31 Princeton Univ. Press, Princeton, NJ 1985.

13. F.C. Kirwan, Partial desingularisations of quotients of non-singular varieties and their Betti numbers. Ann. Math. 122 (1985) 41-85.

14. J. Kollar. Rational curves on algebraic varieties. Ergebnisse der Mathematik und ihrer Grenzgebiete, 32. Springer-Verlag, Berlin, 1996.

15. C. Okonek, M. Schneider and H. Spindler. Vector bundles on complex projective spaces. Progress in Mathematics, 3. Birkhauser, Boston, Mass., 1980.

16. R. Piene. Degenerations of complete twisted cubics. Enumerative geometry and classical algebraic geometry (Nice 1981), Progr. Math. 24, Birkhäuser, 1982.

17. R. Piene and M. Schlessinger. On the Hilbert scheme compactification of the space of twisted cubics. Amer. J. Math. 107 (1985), no. 4, 761-774.

18. C. Simpson. Moduli of representations of the fundamental group of a smooth projective variety. I. Inst. Hautes Etudes Sci. Publ. Math. No. 79 (1994), 47-129.

19. M. Thaddeus. Geometric invariant theory and flips. J. Amer. Math. Soc. 9 (1996), 691-723.

20. I. Vainsencher and F. Xavier. A compactification of the space of twisted cubics. Math. Scand. 91 (2002), no 2, 221-243.

Department of Mathematics, Seoul National University, Seoul 151-747, Korea

E-mail address: dragon10@snu.ac.kr

Department of Mathematics and Research Institute of Mathematics, Seoul National University, Seoul 151-747, Korea

E-mail address: kiem@math.snu.ac.kr 